University of Rhode Island

DigitalCommons@URI

Civil \& Environmental Engineering Faculty

Publications

Civil \& Environmental Engineering

$9-5-2020$

\title{
Strain sensing efficiency of hierarchical nano-engineered smart twill-weave composites: Evaluations using multiscale numerical simulations
}

Sumeru Nayak

Sumanta Das

University of Rhode Island, sumanta_das@uri.edu

Follow this and additional works at: https://digitalcommons.uri.edu/cve_facpubs

The University of Rhode Island Faculty have made this article openly available.

Please let us know how Open Access to this research benefits you.

This is a pre-publication author manuscript of the final, published article.

Terms of Use

This article is made available under the terms and conditions applicable towards Open Access Policy Articles, as set forth in our Terms of Use.

\section{Citation/Publisher Attribution}

S. Nayak, S. Das, Strain sensing efficiency of hierarchical nano-engineered smart twill-weave composites: Evaluations using multiscale numerical simulations, Composite Structures. 255 (2021) 112905.

https://doi.org/10.1016/j.compstruct.2020.112905.

This Article is brought to you for free and open access by the Civil \& Environmental Engineering at DigitalCommons@URI. It has been accepted for inclusion in Civil \& Environmental Engineering Faculty Publications by an authorized administrator of DigitalCommons@URI. For more information, please contact digitalcommonsgroup@uri.edu. 


\title{
Strain sensing efficiency of hierarchical nano-engineered smart twill-weave composites: Evaluations using multiscale numerical simulations
}

\author{
Sumeru Nayak ${ }^{1}$, Sumanta Das ${ }^{2 *}$ \\ ${ }^{1}$ Graduate Student, Civil and Environmental Engineering, University of Rhode Island, Kingston, \\ RI 02881, United States \\ $2^{2}$ Assistant Professor, Civil and Environmental Engineering, University of Rhode Island, \\ Kingston, RI 02881, United States, Email: sumanta_das@uri.edu (corresponding author) \\ https://doi.org/10.1016/j.compstruct.2020.112905
}

\begin{abstract}
This paper evaluates the strain-sensing ability of a nanoengineered hierarchical twill weave composite using multiscale numerical simulations. Piezoresistivity is incorporated in such composite by introducing carbon nanotubes (CNT) in the polystyrene (PSS) matrix so as to form a percolating microstructure. The glass fiber twill weave, which itself contains CNT-modified PSS matrix inside the yarns, is coated with thin film of such piezoresistive matrix to obtain the smart composite configuration. The methodology, presented in this paper, captures the hierarchical intricacies at multiple length scales and implements various mechanical damage mechanisms at subsequent interactive length scales as well as consequent electrical responses so as to yield macroscopic electromechanical response. The simulated responses show excellent correlation with experimental observations signifying the efficacy of the simulation methodology. Such a detailed multiscale approach can provide valuable insights towards tuning of structural hierarchies at multiple length scales for efficient design of smart woven laminated composites.
\end{abstract}

Keywords: Smart hierarchical composites; twill-weave composite; multiscale numerical simulations; micromechanics; Piezo-resistivity; Strain sensing 


\section{INTRODUCTION}

Textile composites are widely used in aerospace, astronautics, marine, automotive and off-shore applications owing to their lightweight nature and exceptional mechanical performance [1,2]. Unlike traditional unidirectional laminated composites which are susceptible to inter-laminar damages, the woven fabrics offer enhanced through-thickness reinforcement thus significantly enhancing the damage tolerance. A long thriving knitting industry and the relative ease of fabrication has catapulted cost-effective plain/twill weaves among the most widely used composites in the modern era. However, with the ever-expanding domain of applications of such fiber reinforced composites, a variety of unforeseen circumstances have appeared including material defects, manufacturing errors, environmental-induced degradation, excessive loading, fatigue amongst others that can cause significant damages in forms of delamination, matrixcracking, inter-laminar fracture, debonding and their combinations which can lead to catastrophic structural failure $[3,4]$. The intricacy of such damage mechanisms in a heterogenous structural material such as fiberreinforced woven polymers pose a tremendous challenge in damage detection. The damages that initiate at micron scale between fibers and matrices or laminae are imperceptible to visual inspection and macroscale sensors like strain gauges, transducers and accelerometers. Emerging sensors like infrared thermometers [5], ultrasonic [6] and fiber optic sensors [7] can enable damage detection, albeit increasing complications. For instance, fiber optic sensors are susceptible to damage rendering vulnerable spots in polymer composites [8]. On the other hand, transducers and ultrasound sensors are susceptible to reduced sensitivity owing to electromagnetic interference resulting in low signal to noise ratios $[9,10]$. This paves the way for development of intrinsic sensors that can enable continuous monitoring while offering superior performance brought about via incorporation of nano-sensors like CNTs which are multifunctional in nature $[10,11]$. Toward that end, nano-engineered composites with CNT-incorporated thin films deposited on fiber weaves have been demonstrated in an experimental study [10] for strain sensing and damage detection. Such CNT-incorporated smart textile composites are reinforced by interlaced fibers thus offering superior mechanical performance owing to consistent through-thickness properties at reduced manufacturing cost. The assembly process in such fabrication processes $[10,12]$ involves layer by layer technique (LbL) and surface modified fibers have been used in such composites to enhance CNT dispersion. Such design and manufacturing technique ensure piezo-resistivity of composites with reversible strain sensing and sufficient sensing-sensitivity. The mechanisms governing the strain sensitivity of such smart weave are the mechanically induced deformations that alter CNT positions or induce damage in matrix or interface thereby varying the overall current distribution in the microstructure. Strain sensing ability of such smart textile composites has been established through experimental evaluation $[10,13]$. While the previous studies evaluated the performance of such composites experimentally, this study assimilates a finite element-based 
framework that entails multiple hierarchical length scales toward obtaining macroscopic electro-mechanical response with a view to enable multiscale simulation-based design of such smart composites. There are various numerical studies available in the literature that account for uncoupled electrical or mechanical property prediction of nanocomposites with CNT modifications [14,15]. Simulation of strain sensing behavior of CNT-modified thin films has also been reported [13]. In the light of previous published literature, the uniqueness of the current study lies in its ability to incorporate the coupled electromechanical responses at various interactive length scales to account for the nano-engineered hierarchical composite while offering the flexibility of application in various weaves with different geometrical or strength characteristics. This is achieved by an integration of the various length scales to form a holistic framework capable of capturing piezo-resistive characteristics in a sequential multiphysics framework that can even be extended with thermomechanical characteristics [16], environmental degradation [17] or piezoelectric effects [18]. Such an approach offers robustness in its ability to incorporate nanoscale modifications that can significantly alter thin film characteristics thereby altering the sensing efficiency of the smart weave composites. Overall, the multiscale numerical simulations, presented in this paper, can potentially facilitate efficient material design of hierarchical nano engineered smart twill weave composites for a wide range of applications.

\section{MULTISCALE ELECTRO-MECHANICAL RESPONSE OF SMART TWILL WEAVE COMPOSITES}

Smart hierarchical textile composites with wavy yarns are inherently heterogenous in nature with a complex microstructure that necessitates computational homogenization at multiple length scales towards prediction of effective laminate properties ranging from stiffness to conductivity. Towards that end, microscopically heterogenous volumes are represented as unit cells at various scales. These unit cells are thereafter used to obtain homogenized properties by analytical and computational techniques. While analytical techniques $[19,20]$ are quick and nimble on resources, those suffer from an oversimplification of heterogeneity often resulting in inadmissible predictions. Computational techniques [21,22], on the other hand, are accurate and can mimic the intricate microstructure involved in such heterogenous composites only being limited by computational expense. The multiscale computational homogenization essentially associates a heterogenous unit cell to every Gauss point of the virtual macro-homogenous structure at subsequent length scales. The robustness of the approach lies in its ability to capture both physical and geometric evolution at each scale resulting in a homogenized constitutive relation at the macroscopic length scale. This is achieved by solving a boundary value problem on a unit cell with consistent boundary conditions obeying HillMandel principle [23]. The discretized system of equations for a field $\psi$ (displacement for mechanical; electric field for electrical response) can be written in terms of the standard FE matrix $\boldsymbol{K}$ (stiffness for 
mechanical; conductivity for electrical), vector of Lagrange multipliers $\lambda$ and vector of field values $\boldsymbol{\psi}$ (vector of displacement values for mechanical; vector of electric field values for electrical) for any $\Omega \subset \mathrm{R}^{3}$ of the unit cell as follows [17]:

$$
\left[\begin{array}{cc}
\boldsymbol{K} & \boldsymbol{C}^{T} \\
\boldsymbol{C} & 0
\end{array}\right]\left\{\begin{array}{l}
\boldsymbol{\psi} \\
\boldsymbol{\lambda}
\end{array}\right\}=\left\{\begin{array}{c}
\mathbf{0} \\
\boldsymbol{D} \nabla \psi
\end{array}\right\}
$$

where $\boldsymbol{C}=\int_{\partial \Omega} \boldsymbol{H} \boldsymbol{N}^{\boldsymbol{T}} \boldsymbol{N} d \partial \Omega, D=\int_{\partial \Omega} \boldsymbol{H} \boldsymbol{N}^{\boldsymbol{T}} \boldsymbol{X} d \partial \Omega$ with $\boldsymbol{N}$ being matrix of shape functions, $\boldsymbol{X}$ being matrix of spatial coordinates evaluated at Gauss points during numerical integration as guided by number of degrees of freedom and $\boldsymbol{H}$ matrix reflects the boundary condition of the unit cell which assigns admissible distribution of traction forces on the boundary. For the periodic boundary conditions adopted in the mechanical study, the tractions are anti-periodic and $\boldsymbol{H}$ matrix on opposite faces are $\boldsymbol{H}^{+}=-\boldsymbol{H}^{-}$. Sample $\boldsymbol{H}$ and $\boldsymbol{X}$ matrices are available in [24,25]. Having solved the system of equations, the homogenized response $\boldsymbol{p}^{\boldsymbol{h}}$ (stress for mechanical; electric flux for electrical) of a unit cell with volume $V$ can be obtained by $\left(D^{T} \lambda\right) / V$. It is to be noted that the number of Lagrange multipliers $\lambda$ per node is equal to the number of degrees of freedom of the node. The aforementioned method enable computation of the homogenized response of the unit cell which under various conditions of field gradient can enable computation of the complete constitutive behavior at the subsequent scale. This is computed by $\overline{\boldsymbol{K}} \nabla \psi$ where $\overline{\boldsymbol{K}}=\boldsymbol{p}^{\boldsymbol{h}}$ subject to various field gradients. The computational homogenization approach described herewith enables computation of the electromechanical responses [26] of nano-engineered matrix incorporated textile composites at interactive length scales described hereafter, thus capturing the inherent heterogeneity in such composites. The interactive length scales are scale-separated (implying length scales are significantly separated) thereby enabling the application of first order computational homogenization described above.

The multiple length scales involved in a hierarchical smart twill weave composite is shown in Figure 1. The hierarchical smart composite, considered in this study for computational evaluation, contains CNTincorporated PSS matrix and glass fiber weave. Thin film of CNT-PSS matrix surrounds the weave as coating which yields piezoresistive interactions in the composite. The LbL assembly technique for fabrication of thin films, which is adequately described in the literature [11,12], proceeds with application of bilayers on the substrate. This study adopts a configuration containing 29 bilayers as reported in an experimental study [10]. In order to capture the various mechanical and electrical phenomena in the smart hierarchical textile composite, the scale-separated computational homogenization approach adopts three interactive lengths scales as shown in Figure 1. 


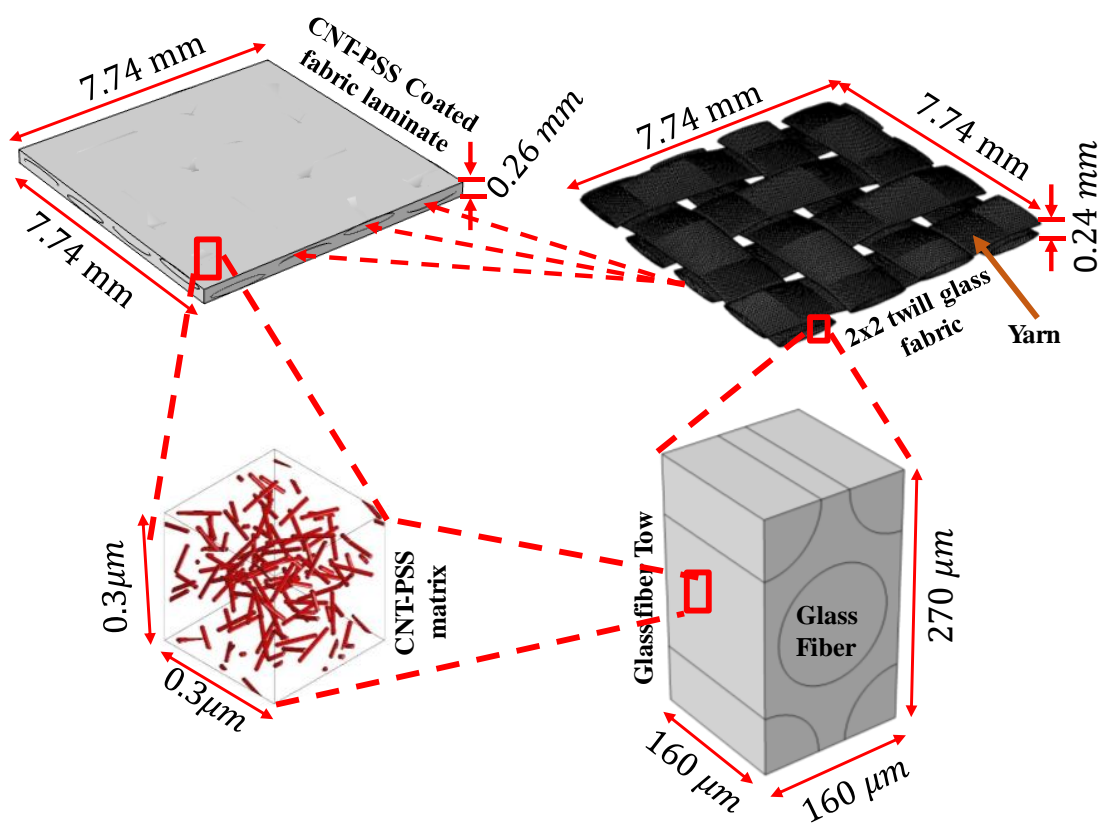

Figure 1. Structural hierarchies in the smart textile weave composite

The macro-scale laminate is represented by a unit cell with a wavelength of the twill fabric. The yarns in the fabric are represented by a hexagonal unit cell representing the fiber arrangements in the tow. The matrix in the coated fabric is itself a composite with CNT-impregnated PSS. The representative unit cells of the weave and tow are benefited from the periodic characteristics of the weave architecture and the fiber distribution in the tows. On the other hand, the CNT-modified matrix is represented by a representative unit cell that implements periodicity during fiber generation. Such an intricate analysis necessitates accurate geometric parameters and fiber fractions at each scale as described hereafter. The forthcoming sub-section first evaluates electromechanical behavior of CNT-PSS nanocomposite matrix which serves as input for macroscopic electromechanical response prediction for smart twill laminated composite. The multiphysical approach towards prediction of electromechanical behavior follows a robust sequential strategy, as adopted in [27] whereby the mechanical deformations are captured in a deformed mesh which is remeshed and used as the starting geometry for electrical simulations. Thereafter, intrinsic electrical properties of the constituents are assigned, and effective electrical response is obtained. The supplementary document includes technical details about mesh generation, mesh convergence studies, RVE size study and software used in the study. 


\subsection{Electromechanical response of CNT-PSS nanocomposite matrix}

\subsubsection{Geometry generation and unit cell}

In the CNT-PSS unit cell, a random 3D network of CNT fillers is generated, the distribution of which is obtained from [15], as shown in Figure 2 (a).
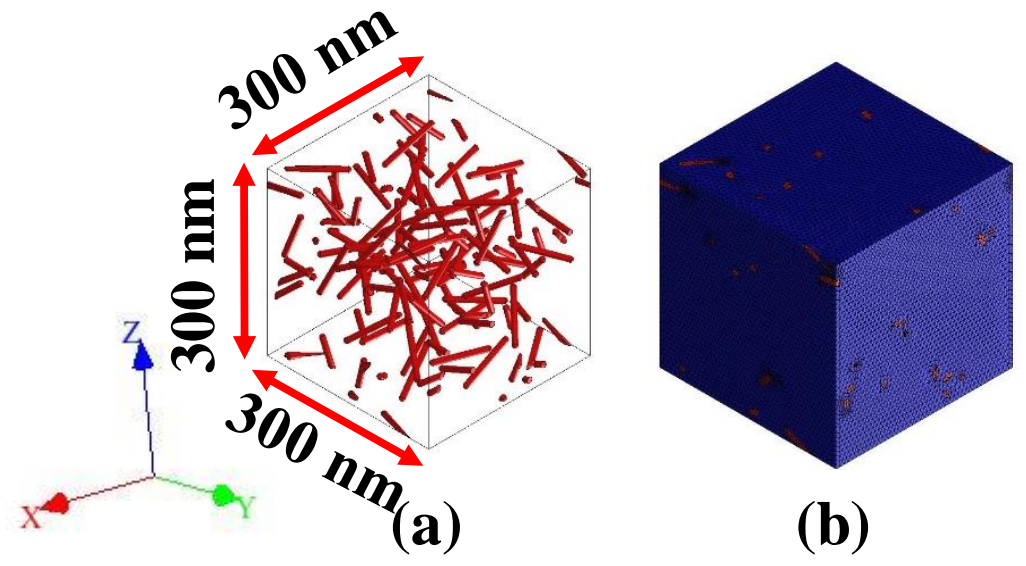

Figure 2. (a) Unit cell representing CNT-PSS and (b) meshed unit cell

CNTs are considered straight solid cylinders with a radius $2 \mathrm{~nm}$. Their length follows a gaussian distribution with a mean value of $100 \mathrm{~nm}$ and a standard deviation of $20 \mathrm{~nm}$. Size distribution of CNTs is adopted from the literature [15]. The generated RVEs have a size of 300nm edge length. The choice of RVE size is governed by a size sensitivity study for elastic property prediction along orthogonal directions, as detailed in the supplementary document (Section C). Such size of RVEs have been shown to be representative in the literature [15]. A boundary wall conditioned approach, as demonstrated in [28], is adopted for this study that involves implementation of a cut-off boundary condition. Such cut-off boundary condition locally trims the fillers protruding the boundary of the unit cell followed by its translation into a respective position on the opposite face. This ensures material periodicity whereby the fillers exceeding the boundary of the unit cell are trimmed and the surplus is placed at the opposite boundary as if the unit cell is a part of a larger set of unit cells. The random microstructure with CNTs is generated by an iterative process whereby random inclusions are seeded and then subjected to a growth rate resulting in the desired particle size distribution. The positions of the inclusions are updated with a constant velocity for every time increment and thereafter checked for overlap. The time increment at every step is adopted to be minimum of those corresponding to every possible colliding inclusion pair. The algorithm proceeds with assignment of updated positions resulting from collision and growth of inclusions in the bounding box at every step only to be terminated at 
the target volume fraction of 0.038 . Such target volume fraction of CNTs has been shown to form percolating microstructure in [10]. The detailed formulations for unit cell generation are mentioned in $[17,27]$. The final step involves the implementation of boundary wall approach, as described earlier, to achieve periodicity. Thereafter, the RVE is meshed (Figure 2(b)) and periodic boundary conditions (PBC) are implemented by constraining nodes on parallel surfaces. A mesh convergence study was performed and a tetrahedral mesh with 1642270 elements was chosen beyond which any further mesh-refinement resulted in insignificant changes in the results. A consistent mesh is obtained at the material boundaries (between CNT fibers and the matrix) following a virtual triangle inversion scheme, as elaborated in Section A of the supplementary document. A. The zero-thickness cohesive elements are generated at the interface by a set of duplicate dummy nodes. The details on mesh generation, PBC application and mesh convergence study can be found in the supplementary document (Section A and B). Prescribed displacement $(\delta)$ is applied along $\mathrm{x}$ direction for tensile simulations. For shear loading, relative displacements (equaling $\delta / 2$ ) are imposed on faces normal to $\mathrm{z}$. The formulations for PBC are adequately described in the authors' previous publications $[27,29,30]$. Geometry generation, meshing and implementation of PBC are done using a python script and the analysis is performed using commercial ABAQUS ${ }^{\mathrm{TM}}$ software with cohesive elements for the interface and solid elements for matrix and fibers. The forthcoming sub-sections evaluate the effective mechanical and electromechanical behavior of CNT-PSS matrix.

\subsubsection{Mechanical behavior of polymer nanocomposite}

\subsubsection{Matrix behavior}

Constitutive equations for describing nonlinear material characteristics of damaged glassy polymers are adopted from [31]. Assuming an isotropic material, the uniaxial behavior of PSS matrix is defined using modified Bodner-Partom (BP) model [31,32]. The BP model [32] defines plastic flow as a correlation between the stress $\boldsymbol{\sigma}$ (its deviatoric component being denoted by $\boldsymbol{\sigma}^{\prime}$ ) and the effective plastic strain $\boldsymbol{p}$ as mentioned in Equation 2.

$$
\boldsymbol{\sigma}=\left(Z_{1}+Z_{2}\right) g(\boldsymbol{p})
$$

where $Z_{1}$ and $Z_{2}$ are internal variables for hardening and softening respectively governed by the plastic work and $g(\boldsymbol{p})$ is a rate dependent functional (rate being governed by parameter $n$ ) defined in terms of $D_{0}\left(=10^{-4}\right)$ for glassy polymers as follows.

$$
g(\boldsymbol{p})=\left(\frac{\sqrt{3}}{2 \mathrm{D}_{0}} \boldsymbol{p}\right)^{1 / 2 n}
$$


In order to obtain the parameters $Z_{1}, Z_{2}$ and $n$, the limiting values of the solution variables are determined from experimental stress-strain observations followed by suitable fitting methods as described in [31]. At the peak stress where the plastic strain $\boldsymbol{p}$ equals total strain, the value of $Z_{2}$ becomes zero (no softening) while the value of $Z_{1}$ is denoted as $Z_{1_{0}}$ initial hardening value). In the current scope of study, the rate dependence of the materials is limited to quasi-static loading which leads to a safe consideration of $n$ being 9.2 [31] thus characterizing the hardening parameter $Z_{1_{0}}$. As observed from stress-viscoplastic strain relationships of glassy polymers, the work hardening rate exhibits a bilinear behavior with stress which can be described by two hardening parameters $m_{1}$ and $m_{2}$. This observation leads to the following relations between the hardening and softening variables $Z_{1}$ and $Z_{2}$ respectively with the plastic work $W^{p}(=$ $\left.\int_{t_{0}}^{t} \boldsymbol{\sigma} \boldsymbol{p} d t\right)$

$$
\begin{gathered}
Z_{1}=(1-\alpha) Z_{1_{0}}+\alpha Z_{1_{0}} \exp \left(m_{1} W^{p} / Z_{1_{0}}\right) \\
Z_{2}=Z_{2 s}\left[1-\exp \left(-m_{2} W^{p}\right)\right]
\end{gathered}
$$

where $Z_{1_{0}}$ is the initial value of $Z_{1}$ (obtained from previous step at yield stress), $Z_{2 s}$ is the saturation value of the softening variable $Z_{2}$. A close observation of the bilinear work hardening rate and stress relationship for glassy polymers shows zero work hardening rate at a value of stress equaling plateau stress from which $\alpha$ can be obtained around 0.28 for such materials. At the plateau stress, the plastic work is denoted by $W_{p}^{p}$ which is used in Equation 4. The slopes of the bilinear relationships (bilinearly fitted between workhardening rate and stress) are $m_{1} / Z_{1_{0}}$ and $m_{2}$. The saturation value of the softening variable is defined by Equation 6.

$$
Z_{2 s}=\frac{1+{ }^{\gamma_{1}} / m_{1} g(\boldsymbol{p})}{Z_{1_{0}}^{-1}+m_{2} / m_{1} \exp \left(-\left(m_{2}+\frac{m_{1}}{Z_{10}}\right) W_{p}^{p}\right)}
$$

where $\gamma_{1}$ is the work hardening rate of the positive arm of the bilinear relationship at zero stress.

In order to characterize damage in glassy polymers, yields surfaces are defined in terms of normalized effective and hydrostatic stresses [33,34]. In the current scope of study, the PSS matrix is considered to be defect free. Once damage sets in such a material, the deviatoric inelastic strain $\boldsymbol{p}^{\prime}$ correlates with post damage stress $\boldsymbol{\sigma}_{d}$ (its deviatoric component being $\boldsymbol{\sigma}_{d}^{\prime}$ ) as per Equation 7 (Note that prior to damage initiation, $\boldsymbol{\sigma}_{d}^{\prime}=\boldsymbol{\sigma}^{\prime}$ ).

$$
\boldsymbol{p}^{\prime}=(1-f) \sigma_{e} \boldsymbol{p}\left(\boldsymbol{\sigma}_{d}: \frac{\partial \phi_{p}}{\partial \boldsymbol{\sigma}_{d}}\right)^{-1} \boldsymbol{\sigma}_{d}^{\prime}
$$




$$
p^{h}=(1-f) \sigma_{e} \boldsymbol{p}\left(\boldsymbol{\sigma}_{d}: \frac{\partial \phi_{p}}{\partial \sigma_{d}}\right)^{-1} q_{1} q_{2} \frac{f}{3} \sigma_{e} \sinh \left(\frac{3}{2} q_{2} \frac{\sigma_{d}^{h}}{\sigma_{e}}\right)
$$

where $q_{1}, q_{2}$ are the internal variables characterizing the distortion phenomena, $f$ is the void fraction, $\sigma_{e}$ is the equivalent stress $\left(=\sqrt{3 / 2 \sigma^{\prime}: \sigma^{\prime}}\right)$ and $\phi_{p}$ is the inelastic flow potential of the material. Thus, the inelastic flow rule as quantified by the aforementioned parameters is defined as follows:

$$
\boldsymbol{p}=\frac{2}{3}(1-f) \lambda \sigma_{e}^{2}\left(\boldsymbol{\sigma}_{d}^{\prime}: \boldsymbol{\sigma}_{d}^{\prime}+q_{1} q_{2} \frac{f}{3} \sigma_{e} \sinh \left(\frac{3}{2} q_{2} \frac{\sigma_{d}^{h}}{\sigma_{e}}\right) \boldsymbol{\sigma}_{d}: \mathbf{I}\right)^{-1}\left(\boldsymbol{\sigma}_{d}^{\prime}+q_{1} q_{2} \frac{f}{3} \sigma_{e} \sinh \left(\frac{3}{2} q_{2} \frac{\sigma_{d}^{h}}{\sigma_{e}}\right) \mathbf{I}\right)
$$

where while the parameter $\lambda$ relates the inelastic strain and deviatoric stress prior to damage initiation as per Equation 10.

$$
\lambda=\frac{3}{2} \frac{p}{\sigma_{e}}
$$

The damage in the material proceeds with void growth that result from void nucleation under loading conditions. A simplified model adopted from [31] captures the growth of voids resulting in damage propagation. The rate of void volume fraction growth denoted by $\dot{f}$ is given by the following Equation.

$$
\dot{f}=3(1-f) p^{h}+\frac{f_{N}}{s \sqrt{2 \pi}} \exp \left[-\frac{1}{2}\left(\frac{p-e_{N}}{s}\right)^{2}\right] p
$$

where the constant $f_{N}$ denotes the void volume fraction resulting from void nucleation, the normal distribution of which has a mean of $e_{N}$ and a standard deviation of $s$. In the current scope of study, the distortion phenomena governing the damage progress in polystyrene are adopted from calibrated models of such glassy polymers which are assumed to be defect-free initially, as mentioned in [31]. While the input Young's modulus and Poisson's ratio for PSS matrix are adopted as $2.4 \mathrm{GPa}$ and 0.38 [35] respectively, the identified parameters, adopted from [31] for PSS matrix are tabulated in Table 1. For CNT, adopted Young's modulus and Poisson's ratio are $500 \mathrm{GPa}$ and 0.3 respectively [36].

Table 1. List of parameters for the PSS matrix with corresponding units adopted from [31]

\begin{tabular}{c|cc} 
Parameter & Unit & Value \\
\hline$D_{0}$ & $s^{-1}$ & $10^{4}$ \\
$n$ & - & 9.2 \\
$Z_{10}$ & $M P a$ & 34.9 \\
$m_{1}$ & - & 20 \\
$m_{2}$ & $M P a^{-1}$ & 12.1
\end{tabular}




\begin{tabular}{c|cc}
$Z_{2 s}$ & $M P a$ & -12.8 \\
$\alpha$ & - & 0.28 \\
$f_{0}$ & - & 0 \\
$\epsilon_{N}$ & - & 0.01 \\
$S$ & - & 0.1 \\
$f_{N}$ & - & 0.22 \\
$q_{10}$ & - & 1.9 \\
$q_{20}$ & - & 1.9 \\
$c$ & - & 0 \\
$N$ & - & 1
\end{tabular}

\subsubsection{Interface behavior}

The interfacial failure of the CNT embedded in the polymer matrix is realized through cohesive finite element method using zero-thickness interface elements [29,37-39]. Such approach has been successfully implemented in the literature [27] for evaluation of electromechanical responses Before onset of damage, the traction-separation cohesive surface behavior is defined as follows:

$$
\sigma=\mathbf{K} \delta
$$

where $\boldsymbol{\sigma}$ is the surface traction, $\mathbf{K}$ is the cohesive stiffness and $\boldsymbol{\delta}$ is the separation of contact surface. Since isotropic cohesive surfaces are implemented in this study, each of the orthogonal directions have equivalent stiffness, strength and fracture toughness. A bilinear traction-separation behavior with linear damage evolution is adopted here [40]. The damage initiation is governed by maximum stress criterion and is characterized by a damage variable that scales linearly from 0 to 1 from damage onset to complete damage, the corresponding respective separations being $\delta^{0}$ and $\delta^{f}$. While $\delta^{0}$ is the separation at which the developed stress equals the strength of the interface, $\delta^{f}$ denotes the separation at failure. The area enclosed by the bilinear traction-separation curve yields the fracture energy $G_{c}$. In this study, the fracture toughness of $85 \mathrm{~mJ} / \mathrm{m}^{2}$ [41] is adopted for the interface whereas the strength of $5 \mathrm{MPa}$ [42] has been incorporated for CNT-PSS unit cell.

\subsubsection{Effective mechanical response of polymer nanocomposite}

The numerical analysis of a representative unit cell containing CNTs in PSS matrix helps to characterize the mechanical behavior of the matrix pockets in subsequent scales. Both tensile and shear loads are applied 
on the unit cell in a displacement-controlled setup. The development of stresses in the virtual microstructure proceeds with concentration pockets around the fibers owing to the stiffness gradient between the inclusion and the glassy matrix. In the three-phase microstructure, the stiff inclusions are embedded in a weak matrix with weaker interfaces. Thus, with increasing strains, the interface damage initiates. The progressive debonding of the fibers with the surrounding matrix is demonstrated in Figures 3(a) and (b) at tensile strains of 0.0145 and 0.0175 respectively. As the debonding progresses at the fiber-matrix interface with increasing strain, the stress in the matrix keeps on increasing loading to onset of void growth in matrix, as captured in Equation 11. This enables quantification of local damage in the matrix. Figures 3(c) and (d) show the progressive damage at tensile strains of 0.02 and 0.03 respectively. A closer observation of the damage patterns reveals strands of local damaged patches, a characteristic of such glassy polymers.

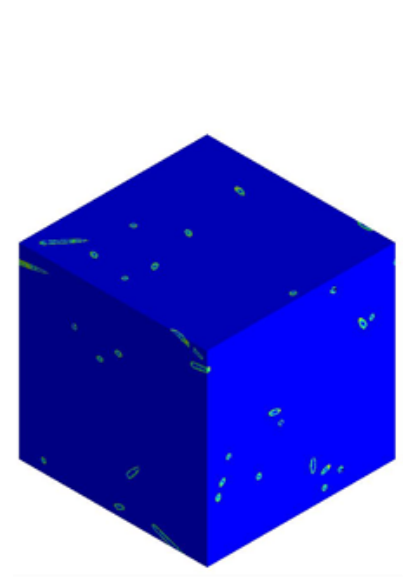

(a)

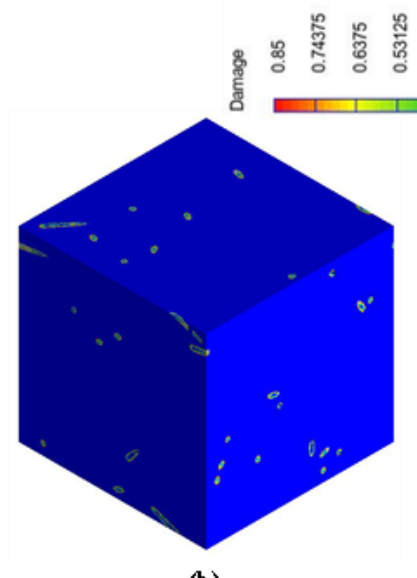

(b)

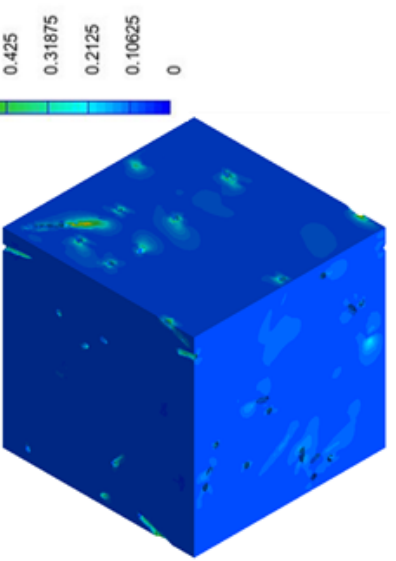

(c)

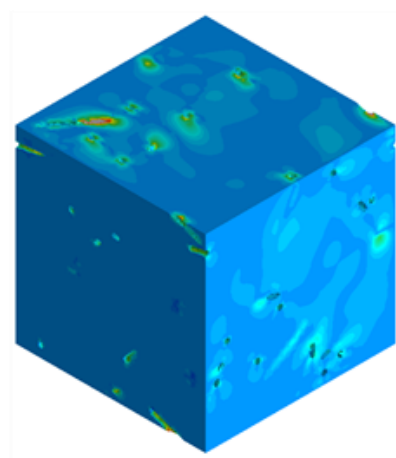

(d)

Figure 3. Progressive debonding and matrix damage at corresponding tensile strains of (a) 0.0145 (b) 0.0175 (c) 0.02 and (d) 0.03 respectively

The tensile behavior of the CNT-PSS nanocomposite is shown in Figure 4. The tensile strength of the matrix as required for subsequent simulation is found to be $24.8 \mathrm{MPa}$ while the tensile modulus is $2.47 \mathrm{GPa}$. As observed from Figure 4, the initial modulus starts degrading once interfacial debonding sets in and it continues until the peak is reached. Beyond the peak, the stiffness degradation is significant implying onset of matrix damage. The plastic behavior of the matrix results in a curved peak. 


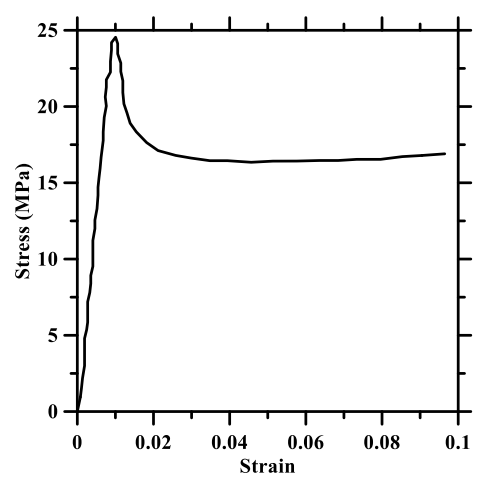

\section{Figure 4. Tensile constitutive response for CNT-PSS nanocomposite matrix}

The incorporation of CNTs in polymers leads to increase in the overall toughness of the composite which can be attributed to pull-out of CNTs besides de-bonding and crack bridging. The overall fracture toughness of the nanocomposite is given as $[43,44]$ :

$$
G_{c}=G_{m}+\sum \Delta G_{i}
$$

where $G_{c}$ is the fracture toughness of the overall nanocomposite, $G_{m}$ corresponds to that of the matrix and $\sum \Delta G_{i}$ denotes the additional fracture toughness brought about by the CNTs. The major contributors to the additional fracture toughness are attributed to the pull-out of CNTs $\left(\Delta G_{\text {pull-out }}\right)$ and the interfacial debonding energy $\left(\Delta G_{d b}\right)$, as demonstrated successfully in [45]. The pull-out contribution of the CNTs is demonstrated in Equation 14.

$$
\Delta G_{\text {pull-out }}=\frac{\tau_{i} V_{p o} l_{e}^{2}}{3 r_{f}}
$$

where $\tau_{i}$ is the interfacial shear stress, $V_{p o}$ is the volume fraction of pulled out fibers, $l_{e}$ is the effective pulled out length and $r_{f}$ is the radius of fiber. The contribution of the debonding to the fracture toughening can be captured by the following Equation [46].

$$
\Delta G_{d b}=\frac{G_{i} l_{f} V_{d b}}{\phi_{f}}
$$

where $G_{i}$ is the interfacial fracture energy between CNT-PSS, $V_{d b}$ is the volume fraction of the debonded fibers, $l_{f}$ and $\phi_{f}$ are the lengths and diameters of fibers.

Figure 5 shows the progressive debonding of the fibers as simulated under tensile strains of 0.017 and shear strain of 0.006 respectively. Having ascertained the debonded volume fractions of the fibers, the enhancement of fracture energy can be computed as described earlier. While the input matrix toughness is 
$0.49 \mathrm{~kJ} / \mathrm{m}^{2}$ [47], the effective fracture toughness of CNT-PSS nanocomposite, obtained from aforementioned simulation, is $0.6 \mathrm{~kJ} / \mathrm{m}^{2}$.
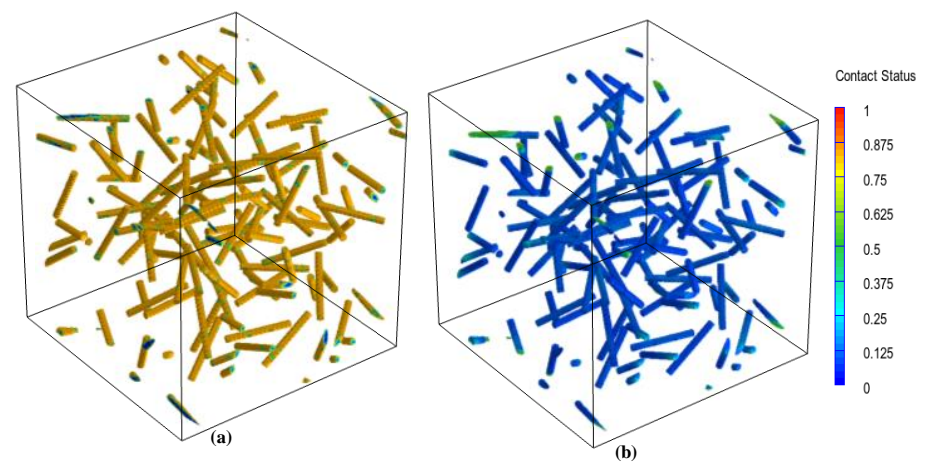

Figure 5. Representative CNT debonding status under: (a) tensile strain of 0.017 (80\% of the fibers are debonded); (b) shear strain 0.006 (35\% of the fibers are debonded)

The input shear toughness for PSS matrix is obtained using a ratio of the shear to normal fracture toughness of 0.54 , which has been successfully adopted for similar glassy matrices in [48]. From the shear simulations, a shear modulus of $1.2 \mathrm{GPa}$ and strength of $6.2 \mathrm{MPa}$ are obtained. The resulting shear toughness, obtained from simulation, for the polymer nanocomposite is $0.3 \mathrm{~kJ} / \mathrm{m}^{2}$. The homogenized responses, obtained here, are used as matrix properties for simulation of smart twill weave composites.

\subsubsection{Effective electrical response of polymer nanocomposite}

\subsubsection{Modification of unit cell geometry to incorporate tunnels}

As mentioned earlier, this study considers a target CNT volume fraction of 0.038 [10] so as to achieve percolation [15]. The interference of the tunneling effect zone is captured in this study by modeling the tunnel zones as domains surrounding the CNTs. Such a procedure of modeling CNTs without intersection while capturing the tunneling zone as domains has been successfully implemented towards percolation prediction in polymer nanocomposites [15]. Owing to deformations arising from loading conditions, the CNTs when separated by a distance less than $0.47 \mathrm{~nm}$ [49] are considered to activate tunnels.

\subsubsection{Establishing percolation}

The percolation theory, introduced in [50], describes the transition from insulator to conductor based on the volume fraction of the conductors. In the current scope of study, the otherwise insulating matrix shows a sudden jump in its overall conductivity once the embedded conductors (here, MWCNTs) form a conducting network. Beyond a certain threshold volume fraction, the conductive pathway is established among the clustering nanotubes which leads to a dramatic increase in the conductivity. The cut-off volume fraction 
that invokes the phase change from insulator to conductor is defined as the percolation threshold. The numerical simulation of the percolating microstructure involves generation of a periodic microstructure of a certain volume fraction of CNTs. Periodic unit cells are highly efficient in prediction of percolation onset as demonstrated in [28]. As mentioned earlier, two nanotubes are considered to be percolating when they are separated by a distance less than a cut-off distance (tunneling distance). Here in this study, the tunnel zones are considered to be of $2 \mathrm{~nm}$ thickness which is consistent with the value reported in the literature [15]. A cut off distance of $0.47 \mathrm{~nm}$ [49] is considered. In a numerical framework, every tube is scanned along its projection in a plane to determine its connectivity with nearest neighbors while checking for percolation along the normal to the plane. The iteration for every nanotube terminates at the co-ordinate where the cut-off distance criterion is satisfied. The percolating path is traced out for each orthogonal direction towards ascertaining the percolation. Figure 6 shows percolation path for considered CNT volume fraction of $3.8 \%$.

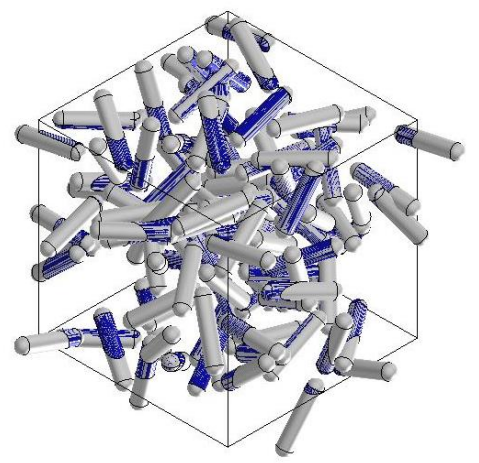

Figure 6. Percolation unit cell showing the connected paths along $X, Y$ and $Z$ directions (grey: connected paths)

\subsubsection{Homogenized electrical response}

In the current scope of study, the Joule heating effect is neglected. Similar strategy has been successfully considered in $[15,49]$. Once the percolating unit cell is obtained, the effective electrical conductivity is computed by the volume average of the electrical field, as mentioned in Equation 16 [15].

$$
\bar{\sigma}=\frac{1}{V} \frac{\int_{V} J d V}{\nabla U}
$$

where $U$ is the potential difference across the faces of the unit cell, $J$ is the local electric current density and $V$ represents the volume. The input electrical conductivities, considered in this study, are $10^{-14} \mathrm{~S} / \mathrm{m}$, $1.37 \times 10^{6} \mathrm{~S} / \mathrm{m}$ and $1.17 \times 10^{-4} \mathrm{~S} / \mathrm{m}$ for the PSS matrix, CNT and tunnel respectively [15].

\subsubsection{Effective electromechanical response of CNT-PSS nanocomposite}


For effective electromechanical response of CNT-PSS nanocomposite, the iterative procedure initiates with a displacement-controlled mechanical loading which results in a deformed configuration and the solution dependent damage progresses in the RVE. A 3D spatial interpolation function is generated for every material domain in terms of the progressive damage variable. This study implements interpolated spatial distribution for defining solution dependent material properties at every domain [51]. A step operation applies proportional decrease in the elemental electrical conductivity depending on the state of damage. This is represented mathematically as follows $[17,27]$ :

$$
\sigma_{i}=(1-D) \sigma_{i-1}
$$

where $\sigma_{i}$ is the current degraded conductivity, $D$ is the mechanical damage variable and $\sigma_{i-1}$ is the conductivity corresponding to previous configuration.

Thus, the effective electrical response of the RVE is obtained for several deformed configurations with varying strain. The electromechanical response of CNT- PSS nanocomposite is evaluated here using fractional change in resistivity (FCR) which is defined as follows [27]:

$$
F C R=\frac{\Delta R}{R_{0}}
$$

where $\Delta R$ is the change in resistivity with varying strain and $R_{0}$ is the bulk resistivity of the material. The correlation of strain with FCR provides a deeper insight into the piezoresitive response of the CNT-PSS thin film which are later used to ascertain the electrical behavior of smart weave under mechanical loads. The current distributions in the RVE corresponding to different strain/damage states are shown in Figure 7. With increasing strain, as the damage progresses several non-conducting pockets start appearing which results in gradual decrease in the overall current flow.

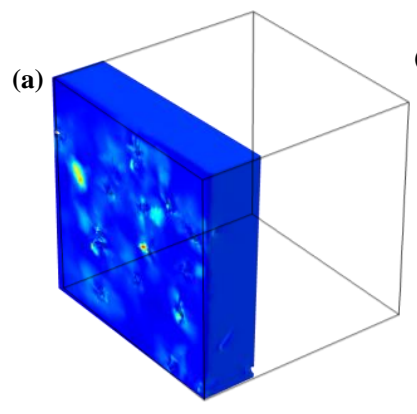

(b)

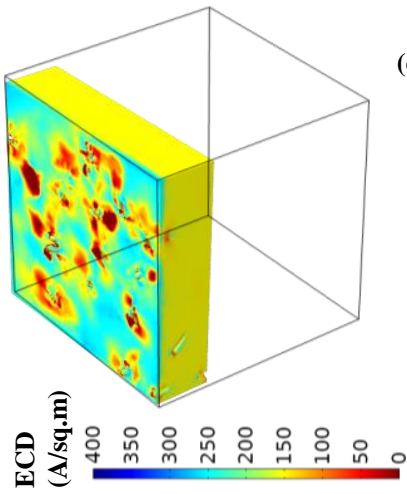

(c)
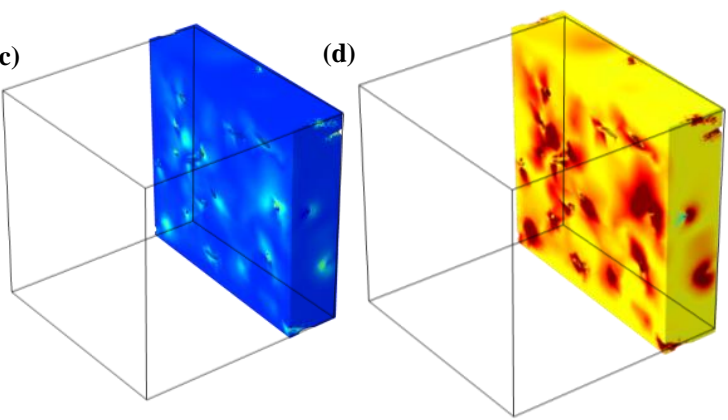

Figure 7. Current density for (a) and (b) front slice and (c) and (d) end slice corresponding to strains of 0.0175 for (a) and (c) and 0.03 for (b) and (d) respectively 
Figure 8 shows the obtained FCR with varying tensile strain for CNT-PSS nanocomposite. While a linear correlation exists in the initial stages (strain up to 0.01), jumps are observed thereafter resulting in nonlinear changes. While the linear changes can be attributed to the piezoresistive characteristics of percolated CNT network in the RVE, the non-linear responses at higher strains result from damages in the interface and matrix. Such mechanically induced phenomena change the current distribution in the RVE which results in increase the overall resistance of the composite (See Figure 4). The piezoresistive response of CNT-PSS nanocomposite, thus obtained, is used later as input matrix property in the analysis of smart weave as described hereafter.

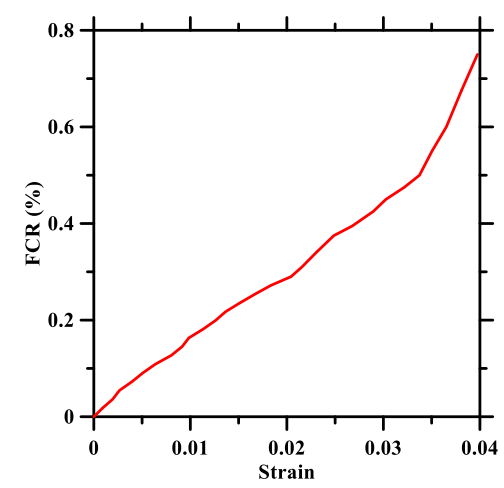

Figure 8. Fractional change in resistivity with strain for CNT-PSS under tensile load

\subsection{Multiscale electro-mechanical response of smart twill weave laminate:}

This section utilizes the homogenized electro-mechanical responses of the CNT-PSS nanocomposite matrix and implements the progressive failure analysis of the hierarchical woven composite. The choice of RVE meshes and the efficacy of PBCs for discontinuous fiber reinforced composites [56] enable accurate prediction of tow behavior. The numerical framework incorporates the squeezing effects of the tows in contrast to often-considered unidirectional behavior. The smart twill weave is subjected to virtual tensile loads and its progressive failure is quantified. Towards that end, micromechanics-based failure criterion is introduced in a multi-scale approach so as to predict the weave damage in the fiber and matrix level. This leads to a damage model that can effectively integrate complex damage mechanisms of the weaves. Having ascertained the deformed configuration, the electrical response is simulated in the discretized temporal domain corresponding to the mechanical states at such instants. This enables a piezoresistive behavior evaluation of the composite laminate in terms of the strain history correlated with the developed stress and fractional change in resistivity.

\subsubsection{Geometries and boundary conditions:}


The representative unit cell $(160 \mu \mathrm{m} \times 160 \mu \mathrm{m} \times 270 \mu \mathrm{m})$ of the periodic fiber arrays within the tows of the weave is shown in Figure 9 (a).

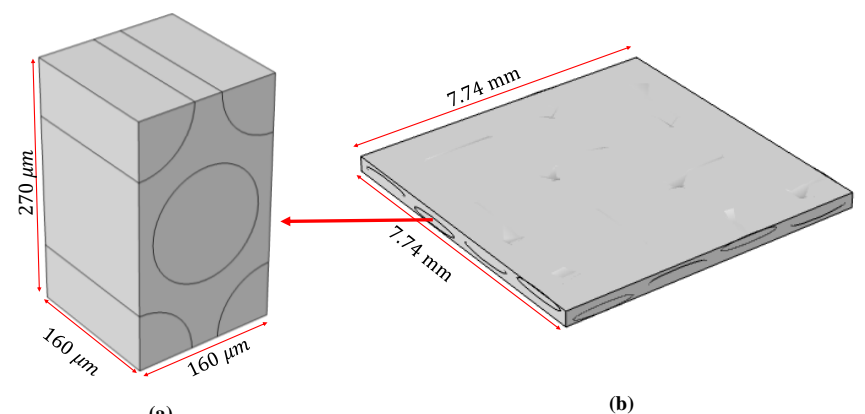

(a)

Figure 9. Unit cell representing: (a) tow of the woven fabric with glass-fiber volume fraction of $52 \%$ (b) CNT-PSS matrix-coated twill weave fabric laminate.

Such representative unit cell was successfully adopted in [52]. The representative unit cell for CNT-PSS matrix-coated twill weave fabric laminate is shown in Figure 9(b). The periodic array for twill fabric are equal in length and width of $7.74 \mathrm{~mm}$. The thickness of the coated laminate is adopted as $0.24 \mathrm{~mm}$. The dimensions are representative of the $2 \times 2$ twill weave with the volume fraction of tow being 0.722 as adopted from [52]. The tows in the unit cell are balanced implying that the warp and weft tows have same waviness ratio and tow volume fraction while those are in full contact [53]. For the CNT-PSS coated woven laminate, the matrix is considered isotropic, with its input mechanical and strain dependent resistivity properties obtained from CNT-PSS simulations (see Section 2.1). The tow behavior is homogenized from the FE analysis of the fiber impregnated matrix unit cell (see Section 2.2.2). Having ascertained mechanical responses from the multiscale framework, the electromechanical responses are obtained by the interpolating strain maps that enable relevant resistivity assignment to the matrix as guided by progressive damage. The weave unit cell is generated in an open-source program TexGen [54] and subsequently solved in ABAQUS $^{\mathrm{TM}}$. The representative unit cells at the tow and weave scales are periodically bounded with individual face nodes being mapped to the opposite face, as enabled by a preprocessor coded in Python.

Adopting the repetitive nature of the unit cells at each scale, periodic boundary conditions are applied. The efficiency of such boundary conditions towards effective property prediction of inclusion embedded systems with various planes of symmetry are detailed in [55]. The relative displacement $\left(u_{i}\right.$ for $\left.i=x, y, z\right)$ for each pair of nodes on the parallel boundary surfaces (represented by point A and point B lying on such faces) of the unit cell are given by the following Equation [52].

$$
u_{i}^{A}-u_{i}^{B}=\epsilon_{a v g} \Delta x_{k}
$$


where $\epsilon_{a v g}$ is the average strain in the unit cell and $\Delta x_{k}$ is the position vector connecting the points on the parallel boundaries. The boundary conditions are implemented by setting a constraint between each pair of nodes on the parallel boundary surfaces. The node pairs are correlated by a preprocessor to ensure the correspondence between such nodes on parallel surfaces. Such an implementation has been demonstrated in [57] to maintain traction and displacement continuity on the parallel surfaces. Further details about conformal mesh generation and PBC implementation are provided in Section A of the supplementary document.

\subsubsection{Simulation methodology for mechanical response of smart twill weave laminate}

The smart twill weave laminate comprises the fiber tows embedded in the resin with a tow-matrix interface. The overall framework followed in this study is elucidated in Figure 10. The laminate analysis commences with obtaining the homogenized properties of the fiber tow and the CNT-modified matrix. While the matrix properties are obtained from randomly generated unit cells (See Section 2.1.2), the homogenized fiber tow properties are obtained from the hexagonal unit cell (see Figure 9(a)). Having ascertained the constituent properties, the macro-scale laminate analysis is initiated. With increasing applied uniaxial tensile strains, the stress responses in the laminate elements are first obtained in each material domain (the tows and coated films with an interface). If the element represents the matrix, the homogenized constitutive response for CNT-PSS nanocomposite (explained earlier in Section 2.1.2.3) is adopted. The damage initiation and propagation in the matrix result in degraded overall stiffness. For the zero-thickness elements representing the tow-matrix interface, interfacial debonding initiation and propagation criteria is implemented as explained later in this paper. When the macro-scale laminate element represents fiber tow, constituent level micromechanics of failure approach is invoked. Such an approach is adopted for fiber tow due to its orthotropic nature and respective failure criteria along different directions. In this approach, at the integration points of the element, the micron-scale analysis involving matrix-glass fiber hexagonal representative unit cell is initiated and the failure status at the matrix and fibers is evaluated. This approach provides direct contribution towards stiffness-degradation of the macro-scale element if damage is initiated in the fibers or matrix within the tow. Thus, the aforementioned approach provides damage status at the matrix, fiber tow and matrix-tow interface which yields homogenized macroscopic tensile stress-strain responses for woven laminated composite. Figure 10 illustrates the framework for mechanical response prediction of woven unit cell. The input properties to the weave unit cell correspond to that of the homogenized tow (Section 2.2.2.1) and homogenized matrix (Section 2.1.2). The progressive debonding in the tow-matrix interface is detailed in Section 2.2.2.2. The tow analysis (Section 2.2.2.3) involves a micromechanical analysis that implements damage in matrix and fiber inside the tow. The thin film analysis involves damage initiation and progression in the matrix (Section 2.2.2.4). The stiffness degradation 
brought about by accumulated damages in the matrix and fiber are thereafter assimilated in the weave unit cell as a continuum damage tensor (Section 2.2.2.5). A mesh convergence study was performed for both fiber tow and the weave unit cell. Mesh convergence was achieved with a tetrahedral volume mesh with 331836 and 2223642 elements (see Section B of the Supplementary document for more details) for the fiber tow (Figure 9(a)) and weave unit cell (Figure 9(b)) respectively. Consistent mesh is obtained at the material boundaries following a virtual triangle inversion scheme, as elaborated in Section A of the supplementary document. The zero-thickness cohesive elements are generated at the material interfaces by a set of duplicate dummy nodes (see Section A of the supplementary document). While the mesh convergence studies are provided in Section B, the details of the software and tools used are provided in supplemental Section D of the supplementary document.

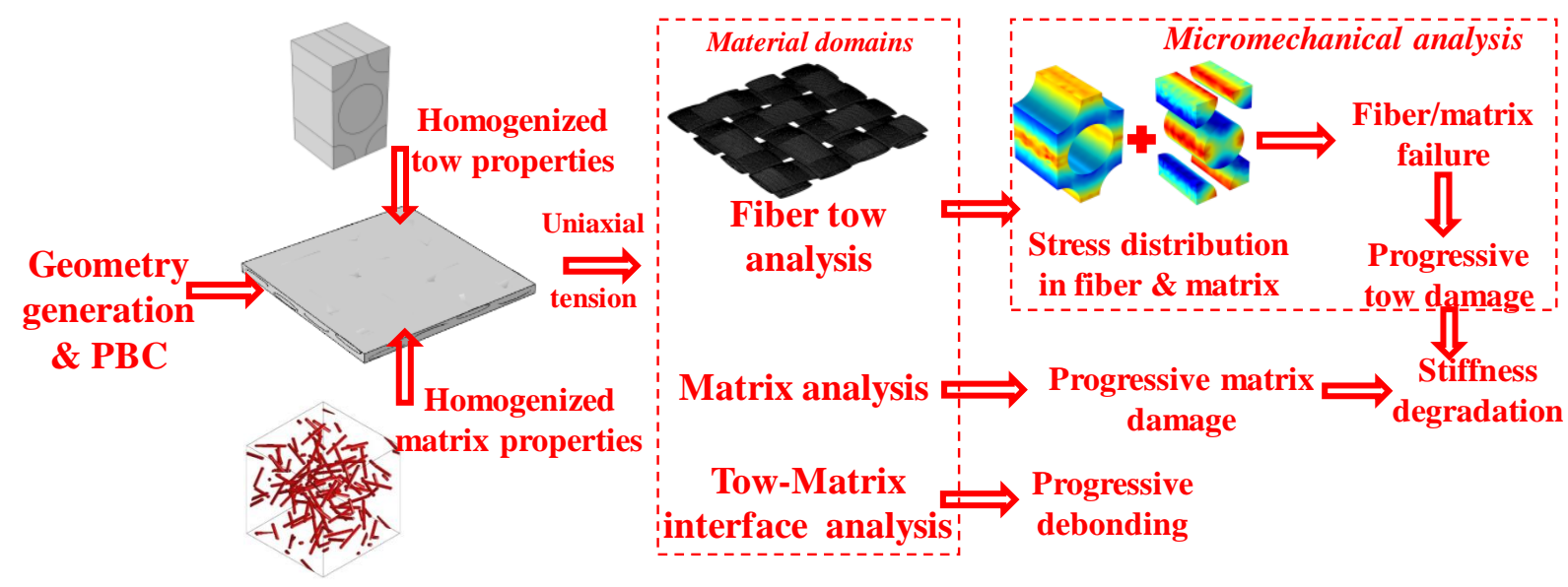

\section{Figure 10. Framework showing the mechanical response prediction of the smart weave unit cell}

\subsubsection{Homogenized tow properties: Input to weave unit cell}

For fiber tow, the unit cell representing the tow (see Figure 9(a)) is bounded periodically and subjected to tensile and shear loadings (similar to Section 2.1.2) to obtain its effective properties. Table 2 lists the obtained homogenized tow properties which are used as input to the macro-scale laminated woven composite analysis.

Table 2: Homogenized tow properties

\begin{tabular}{|c|c|c|c|}
\hline \multicolumn{4}{|c|}{ Homogenized Tow } \\
\hline Parameters & Direction & Units & Value \\
\hline \multirow{2}{*}{$\begin{array}{c}\text { Young's } \\
\text { Modulus }\end{array}$} & Axial & GPa & 36.55 \\
\cline { 2 - 4 } & Transverse & GPa & 8.10 \\
\cline { 2 - 4 } & Out-plane & GPa & 8.10 \\
\hline Shear Modulus & Axial & GPa & 4.12 \\
\hline
\end{tabular}




\begin{tabular}{|c|c|c|c|} 
& Transverse & $\mathrm{GPa}$ & 4.12 \\
\cline { 2 - 4 } & Out-plane & $\mathrm{GPa}$ & 4.12 \\
\hline \multirow{3}{*}{ Poisson's ratio } & Axial & - & 0.3 \\
\cline { 2 - 4 } & Transverse & - & 0.3 \\
\cline { 2 - 4 } & Out-plane & - & -0.016 \\
\hline
\end{tabular}

\subsubsection{Response of Tow-matrix interface}

This section elaborates the behavior of the tow-matrix interface implemented using zero-thickness cohesive elements. Progressive debonding of the tow-matrix interface helps to capture the stress gradients observed near geometric discontinuities in the weave composite. A bilinear traction-separation law is followed to characterize the cohesive elements at the interface of the yarns and the matrix. Before the damage onset, the linear traction separation law enforces an elastic behavior, as represented by Equation 20.

$$
\left(\begin{array}{l}
t_{n} \\
t_{s} \\
t_{t}
\end{array}\right)=\left(\begin{array}{lll}
K_{n} & & \\
& K_{s} & \\
& & K_{t}
\end{array}\right)\left(\begin{array}{l}
\delta_{n} \\
\delta_{s} \\
\delta_{t}
\end{array}\right)
$$

where $t_{i}$ are the tractions, $K_{i}$ are stiffnesses and $\delta_{i}$ are separations of contact surfaces for $(i=$ $n$ for normal and $s, t$ for shear). For numerical convergence, a large value of stiffness is adopted to represent perfect bonding at the interface elements without separation, as demonstrated in [58]. The damage initiation criteria is shown in Equation 21 [59].

$$
\left(\frac{\left\langle t_{n}\right\rangle}{t_{n}^{0}}\right)^{2}+\left(\frac{\left\langle t_{s}\right\rangle}{t_{s}^{0}}\right)^{2}+\left(\frac{\left\langle t_{t}\right\rangle}{t_{t}^{0}}\right)^{2}=1
$$

where $\langle x\rangle \in R$ is defined as $(x+|x|) / 2$.

After damage initiation, the progressive damage follows a BK criterion [52]towards softening behavior characterization as shown in Equation 22.

$$
G_{C}=G_{n C}+\left(G_{s C}-G_{n C}\right)\left(\frac{G_{s}+G_{t}}{G_{s}+G_{t}+G_{n}}\right)^{\eta}
$$

where $G_{C}$ is mixed-mode fracture toughness, $G_{n C}$ and $G_{S C}$ are normal and shear critical toughness of the interface (See Table 3), $\eta$ is a material constant (adopted as 1.45 [59]). The computation of $G_{i}$ (for $i=$ $n, s, t)$ comprises the area under the corresponding traction-separation curve (for normal and shear loads).

The interface damage variable is similarly defined as follows. 


$$
D=\frac{\delta_{m}^{f}\left(\delta_{m}^{\max }-\delta_{m}^{0}\right)}{\delta_{m}^{\max }\left(\delta_{m}^{f}-\delta_{m}^{0}\right)} ; \delta_{m}^{0} \leq \delta_{m}^{\max } \leq \delta_{m}^{f}
$$

where $\delta_{m}^{\max }$ is the maximum value of $\delta_{m}$ (effective separation) throughout the loading history. The effective separation is given by $\left(\delta_{m}=\sqrt{\left\langle\delta_{n}\right\rangle^{2}+\delta_{s}^{2}+\delta_{t}^{2}}\right)$. The effective separation at the onset of damage denoted by $\delta_{m}^{0}=\left(T_{i n t} / K\right)$ is given by the ratio of interfacial strength $\left(T_{i n t}\right)$ and stiffness $(K)$. The mixedmode separation at failure denoted by $\delta_{m}^{f}$ is given by ${ }^{2} G_{C} / K \delta_{m}^{0}$ where $G_{C}$ is computed by B-K equation (see Equation 22). The tractions are continuously updated during the loading process as per the following Equation [59].

$$
\begin{gathered}
t=K \delta \text { for elastic stage }\left(\delta_{m}^{\max }<\delta_{m}^{0}\right) \\
t=(1-D) \bar{t} \text { for softening stage }\left(\delta_{m}^{0}<\delta_{m}^{\max }<\delta_{m}^{f}\right) .
\end{gathered}
$$

where $\bar{t}$ is the traction stress component calculated by linear-elastic traction separation behavior for the current separation displacement. The strength for tow-matrix interface is considered 1.5 times that of the transverse strength of the tow [60]. Thus, the strength is computed as $36 \mathrm{MPa}$ from the yarn properties reported in [61]. The normal fracture toughness for coated glass systems is $0.280 \mathrm{~J} / \mathrm{mm}^{2}$, as determined in [62]. A similar value for normal toughness has been adopted in [59] which reports the shear fracture toughness as $1.45 \mathrm{~J} / \mathrm{mm}^{2}$ which has been adopted in the study. The input interface properties are tabulated in Table 3.

Table 3. Input properties for tow-matrix interface debonding

\begin{tabular}{|c|l|r|}
\hline \multicolumn{3}{|c|}{ Tow-matrix interface } \\
\hline Parameters & Units & Value \\
\hline strength & $\mathrm{MPa}$ & 36 \\
\hline$G_{n}$ & $\mathrm{~J} / \mathrm{mm}^{2}$ & 0.28 \\
\hline$G_{S}$ & $\mathrm{~J} / \mathrm{mm}^{2}$ & 1.45 \\
\hline$G_{t}$ & $\mathrm{~J} / \mathrm{mm}^{2}$ & 1.45 \\
\hline
\end{tabular}

\subsubsection{Mechanical behavior of Tow}

During the tow analysis, a micromechanics-based framework invokes a constituent level response prediction thus facilitating a multiscale analysis. This is brought about by computation of stresses at the fiber and matrix material domains of the hexagonal unit cell representing the tow when macro-stress appears in the tow of the laminate. When the macrostructure is subjected to an external load, the micro-stress at the level of its constituents namely fiber and matrix are characterized in terms of amplification factors at salient points (refer to Figure 11) in a representative unit cell as follows $[52,63,64]$ : 


$$
\boldsymbol{\sigma}=\boldsymbol{M} \bar{\sigma}
$$

where $\boldsymbol{M}$ is the stress amplification factor, $\bar{\sigma}$ is the macro scale stress and $\boldsymbol{\sigma}$ is the micro-scale stress. In order to determine the amplification factor, a periodic representative volume of the fiber and matrix (see Figure 11) is subjected to a FEA with unit traction boundary condition.

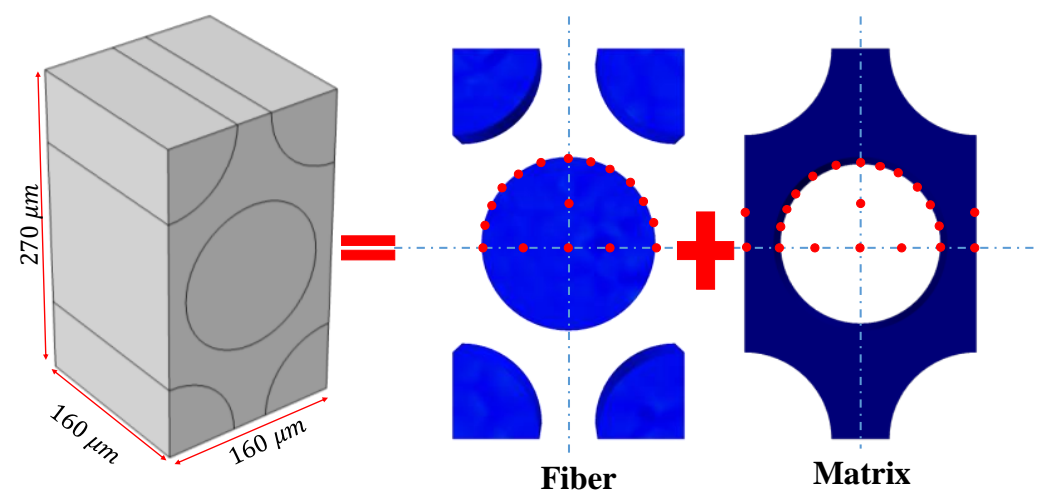

\section{Figure 11. Unit cell representing tow of the woven fabric. The points marked in red are salient points for characterization of micro-stress distribution in fiber (17 points) and matrix (19 points) respectively}

Under a single traction boundary (say $\bar{\sigma}_{i}=1$ ), the corresponding $\sigma_{i}$ is obtained at representative distinct points in the matrix and the fiber (Figure 11). Since $\bar{\sigma}_{i}=1$, the corresponding $M_{j i}=\sigma_{i}$ which suggests that each component of the micro-scale stress at a point represents the amplification factor at that point. An array of judiciously chosen points (17 in fiber and 19 in matrix as shown in Figure 11), as adopted from [63] serve as the key points for efficient characterization of amplification factor of the fiber and matrix. Averaged amplification factors from the selected salient points are adopted for fiber and matrix separately. The methodology enables the computation of stresses at level of constituents (matrix and fiber) when the fiber tows are loaded in the laminate scale. Such a procedure has been successfully implemented towards multi-scale failure analysis of fiber-reinforced composite cylinder [64] and woven composite [52]. Figures 12 (a), (b) and (c) show a schematic representation of the macroscopic stress states while Figures 12 (d), (e) and (f) show the corresponding constituent level stresses (micro-stress distribution). Since the micromechanics framework enables computation of the stresses at the constituent level, the failure initiation and propagation can be carried out at the level of the constituents (matrix and fiber), as described hereafter. 


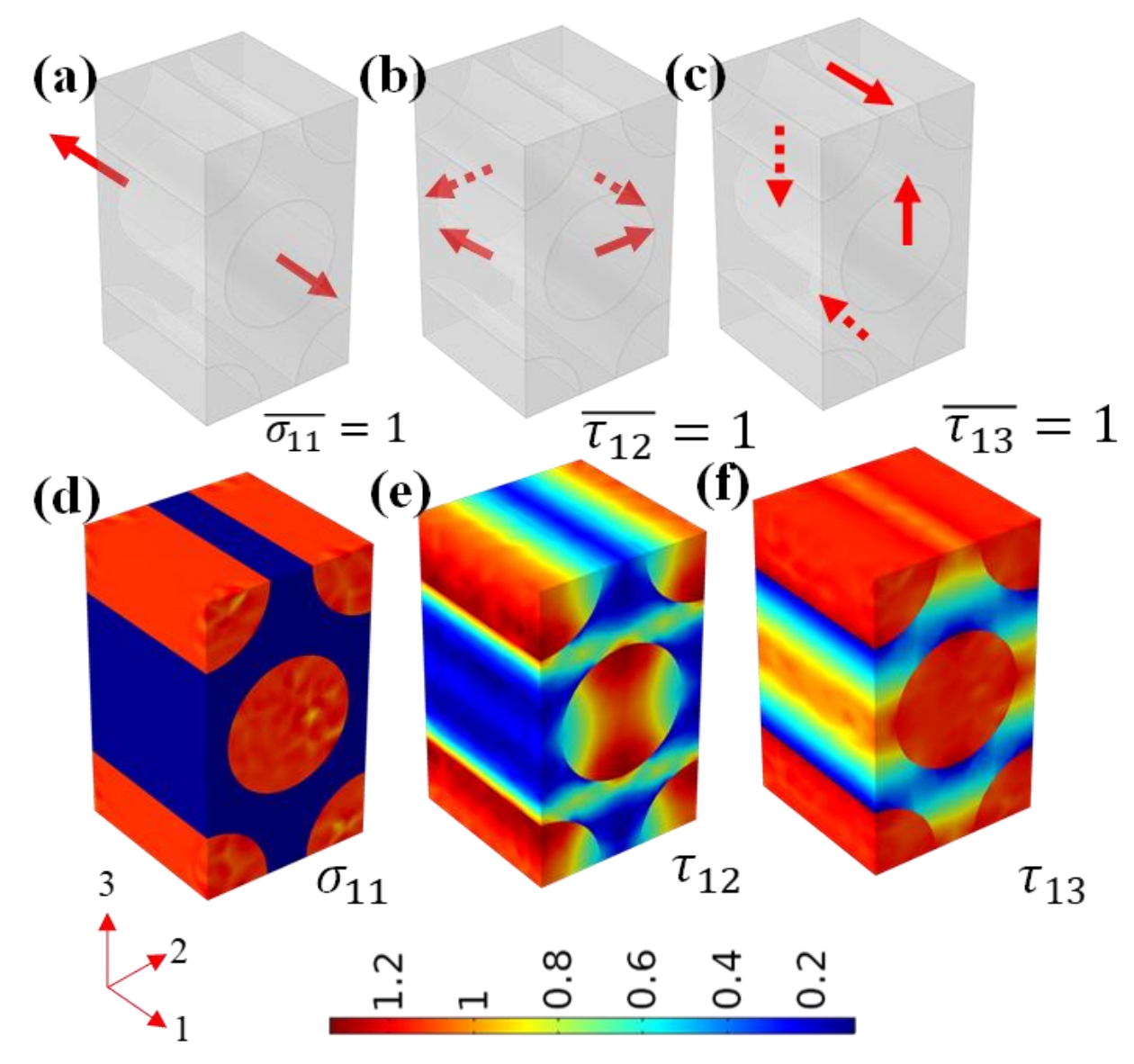

Figure 12. Representative macroscopic stress states $\bar{\sigma}_{11}, \bar{\tau}_{12}$ and $\bar{\tau}_{13}$ set to unity in (a), (b) and (c) respectively; corresponding micro-level stress distributions for $\sigma_{11}, \tau_{12}$ and $\tau_{13}$ (expressed in $\mathrm{N} / \mathrm{m}^{2}$ ) in (d), (e) and (f) respectively

Failure prediction of composites at the constituent level can be brought about by micromechanics of failure (MMF) theory. This translates to the tow behavior whereby almost the entire load is supported by the fibers for longitudinal loads. This justifies the characterization of fiber dominated damage initiation by a noninteracting maximum stress criterion. The fiber failure criterion is given by Equation 27.

$$
{ }^{\sigma_{f}} /_{T_{f}}=1 ;{ }^{\sigma_{f}} / C_{f}=1
$$

where $C_{f}$ and $T_{f}$ are the compressive and tensile strengths of the fibers while $\sigma_{f}$ is the fiber longitudinal stress at the micro scale. The matrix failure initiation criteria is adopted based on von Mises stress at micro level [52]:

$$
\frac{\sigma_{v m}^{m}}{T_{m}}=1
$$

where $T_{m}$ is the matrix tensile strengths, $\sigma_{v m}^{m}$ is the matrix von Mises stress at the micro level. 
This study adopts damage evolution based on an energy equivalence between strain energy dissipated during failure and fracture energy at failure [52]. The following formulations culminating in a damaged stiffness matrix proceed with an iterative computation of the fracture energy of the stressed elements in the matrix and fibers. It is to be noted that the longitudinal and transverse directions in the orthotropic material are represented as $l, t$ and $z$ which are denoted by an index $i$. The fracture toughness $G_{i}$ over a characteristic element length $l$, in purview of the crack band theory can be related to the dissipated energy rate per unit volume $g_{i}$ as follows [65]:

$$
g_{i}=\frac{G_{i}}{l}
$$

where $g_{i}$ is the area under the equivalent stress and equivalent displacement curve $\left(=\frac{1}{2} \sigma_{i, e q} \delta_{i, e q}\right)$. For every component, the equivalent stress and displacement per unit characteristic length are constructed as follows [52,64]:

$$
\begin{gathered}
\delta_{i, e q}=l \epsilon_{i, 11} ; \sigma_{i, e q}=\sigma_{i, 11} \epsilon_{i, 11} / \delta_{i, e q}[\text { for fiber }] \\
\left.\delta_{i, e q}=l \sqrt{\epsilon_{i, n}^{2}+\gamma_{i, s}^{2}+\gamma_{i, t}^{2}} ; \sigma_{i, e q}=\frac{\sigma_{i, n} \epsilon_{i, n}+\tau_{i, s} \gamma_{i, s}+\tau_{i, t} \gamma_{i, t}}{\delta_{i, e q}} \text { [for matrix }\right]
\end{gathered}
$$

where $\epsilon_{i, 11}$ and $\sigma_{i, 11}$ are fiber longitudinal strains and stresses; while for matrix, $\epsilon_{i, n}$ and $\sigma_{i, n}$ are longitudinal strains and stresses respectively; $\gamma_{i, s}, \gamma_{i, t}$ and $\tau_{i, s}, \tau_{i, t}$ are shear strains and stresses respectively along the transverse directions. For characterizing fiber failure, its longitudinal fracture toughness is considered. Meanwhile, a mixed-mode fracture energy B-K criterion [52] is adopted for matrix failure, as shown in Equation 32 [66].

$$
G_{T C}=G_{I C}+\left(G_{I I C}-G_{I C}\right)\left(\frac{G_{I I}}{G_{I}+G_{I I}}\right)^{\eta}
$$

where $G_{I}=\frac{1}{2}\left(\sigma_{n} \epsilon_{n}\right) l ; G_{I I}=\frac{1}{2}\left(\tau_{s} \gamma_{s}+\tau_{t} \gamma_{t}\right) l ; G_{I C}$ and $G_{I I C}$ are mode I and mode II fracture toughness of the matrix, $\eta$ is a material constant. The damage variable based on effective displacement is defined as follows.

$$
d_{i}=\frac{\delta_{i, e q}^{f}\left(\delta_{i, e q}-\delta_{i, e q}^{0}\right)}{\delta_{i, e q}\left(\delta_{i, e q}^{f}-\delta_{i, e q}^{0}\right)} ; \delta_{i, e q}^{0} \leq \delta_{i, e q} \leq \delta_{i, e q}^{f}
$$

where $\delta_{i, e q}^{0}$ and $\delta_{i, e q}^{f}$ are equivalent displacements at damage initiation and complete failure states. For every component (matrix and fiber), the corresponding damage values $d_{i}$ in $l, t$ and $z$ directions help to form the damage tensor $\boldsymbol{D}$ for the components in the woven composite, as described in Section 2.2.2.5. 


\subsubsection{Behavior of Matrix in laminated woven composite}

The thin film/matrix analysis of the woven unit cell proceeds with inputs from the CNT-PSS nanocomposite homogenized response (Table 4). Damage initiation and propagation follow similar formulations as mentioned in Section 2.2.2.3 since the matrix remains the same inside or outside (film) of the tow. The damage values $d_{i}$ (similarly obtained as shown in Equation 33) characterize the stiffness degradation in the woven unit cell, as described in the forthcoming sub-section.

\subsubsection{Damage in macro-scale weave unit cell}

The constituent damages in the matrix and the fiber are assimilated for damage characterization in the representative unit cell of the macro-scale woven composite. Towards that end, the Murukami-Ohno damage model is adopted [52] as shown in Equation 34.

$$
\boldsymbol{D}=\sum D_{i} \boldsymbol{n}_{\boldsymbol{i}} \otimes \boldsymbol{n}_{\boldsymbol{i}}
$$

where $D_{i}$ is the principal damage tensor and $\boldsymbol{n}_{\boldsymbol{i}}$ the principal unit vector (for longitudinal and transverse directions of the orthotropic material). It is to be noted that the ensuing damage in such composites is irreversible which leads to the damage tensor being the maximum damage encountered in the history. Thus, the damage of the fiber tows in the longitudinal direction is considered to be the maximum fiber damage value under longitudinal loading $\left(D_{L}=\max d_{\text {fiber }}\right)$ while the damage for the transverse directions is considered to be the maximum in the matrix $\left(D_{T}=D_{Z}=\max d_{\text {matrix }}\right)$. For the matrix, the tensor assumes an isotropic form with the maximum damage in the matrix corresponding to longitudinal loads. As per [52], the effective stress in damaged configuration is given by:

$$
\sigma^{*}=\frac{1}{2}\left[(\mathbf{I}-\boldsymbol{D})^{-1} \sigma+\sigma(\mathbf{I}-\boldsymbol{D})^{-1}\right]
$$

where $\sigma^{*}$ is the effective stress while $\sigma$ is the actual stress in the damaged configuration. The stiffness matrix in damaged configuration is defined as follows.

$$
\boldsymbol{C}(D)=\left[\begin{array}{rrrrrrr}
d_{1}^{2} C_{11} & d_{1} d_{2} C_{12} & d_{1} d_{3} C_{13} & 0 & 0 & 0 \\
& d_{2}^{2} C_{22} & d_{2} d_{3} C_{23} & 0 & 0 & 0 \\
& & d_{3}^{2} C_{33} & 0 & 0 & 0 \\
& & & d_{4} C_{44} & 0 & 0 \\
& & & & d_{5} C_{55} & 0 \\
& & & & & d_{6} C_{66}
\end{array}\right]
$$


where $C_{i j}$ are the undamaged stiffness matrix coefficients, $d_{1,2,3}=\left(1-D_{L, T, Z}\right), d_{4}=\left(\frac{2 d_{1} d_{2}}{d_{1}+d_{1}}\right)^{2}, d_{5}=$ $\left(\frac{2 d_{3} d_{1}}{d_{3}+d_{1}}\right)^{2}$ and $d_{6}=\left(\frac{2 d_{2} d_{3}}{d_{2}+d_{3}}\right)^{2}$. For the user defined material subroutine, the tangent constitutive tensor in damaged configuration is given by.

$$
\boldsymbol{C}_{\boldsymbol{T}}=\frac{\partial \sigma}{\partial \boldsymbol{\epsilon}}=\boldsymbol{C}(D)+\left(\sum_{L} \frac{\partial \boldsymbol{C}(D)}{\partial D_{L}} \frac{\partial D_{L}}{\partial d_{L}} \frac{\partial d_{L}}{\partial \epsilon}\right): \epsilon
$$

The matrix and fiber input properties are mentioned in Table 4. While the parameters for matrix used in the study are obtained from numerical homogenization of the CNT-PSS unit cell as explained earlier in this paper, the values for glass fiber are adopted from literature $[67,68]$. The formulations are implemented in a user defined subroutine and solved in ABAQUS ${ }^{\mathrm{TM}}$.

Table 4. Matrix and fiber properties

\begin{tabular}{|c|c|c|c|}
\hline Parameters & Units & $\begin{array}{c}\text { Glass } \\
\text { fiber }\end{array}$ & Matrix \\
\hline Young's Modulus & $\mathrm{GPa}$ & 68 & 2.47 \\
\hline Shear Modulus & $\mathrm{GPa}$ & 26.1 & 1.2 \\
\hline Poisson's ratio & & 0.22 & 0.3 \\
\hline Tensile strength & $\mathrm{MPa}$ & 1771 & 24.8 \\
\hline Shear strength & $\mathrm{MPa}$ & - & 6.2 \\
\hline Normal toughness & $\mathrm{kJ} / \mathrm{m}^{2}$ & 3.7 & 0.6 \\
\hline Shear toughness & $\mathrm{kJ} / \mathrm{m}^{2}$ & - & 0.3 \\
\hline
\end{tabular}

\subsubsection{Macroscopic mechanical response of smart twill laminate}

The implementation of the material behavior in the textile composite at interactive scales of the tow and the coated weave enables computation of the tensile response shown in Figure 13 on application of tensile load in the warp direction. The linear elastic behavior is observed up to a strain of 0.005 beyond which the matrix damage initiates. Another change of slope is observed at around a strain of 0.0095 which corresponds to the initiation of debonding of the tow. As the debonding proceeds, the singular tows are stressed owing to localization of stresses due to lack of contact. This leads to damage propagation in the tow at a strain of around 0.015 . Finally, this leads to fiber failure at a strain of around 0.018 following which there is almost an instantaneous drop in stress. 


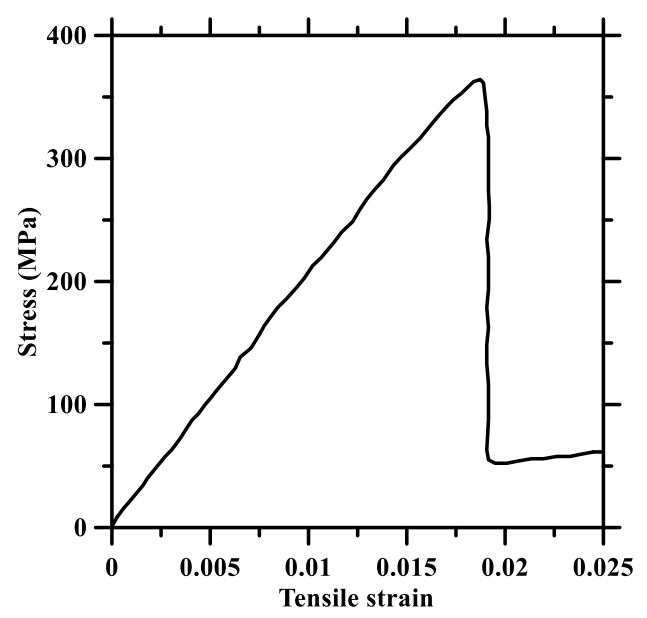

Figure 13. Macroscopic stress-strain response under tensile load applied parallel to weave

\subsubsection{Electromechanical response of smart twill laminate}

For electromechanical response, the conductivity of the glass fiber is considered $2.54 \mathrm{e}-11 \mathrm{~S} / \mathrm{m}[69]$ and the electromechanical response of the coating matrix (CNT-PSS nanocomposite), as detailed in Section 2.1, is adopted to represent variation of electrical conductivity with varying state of strain. When damage initiates, a proportional decrease in the elemental electrical conductivity is considered, similar to the methodology detailed in Section 2.1.4. The piezoresistive nature of the nano-engineered composite as captured by the simulation enables the computation of the FCR. Figure 14 shows the electromechanical behavior of the coated weave. Figures 14 (a-d) show the tows at various states of strain while Figures 14 (e-f) show the damage response of the matrix. With increasing strain progressive damage in the yarns can be clearly observed in both weft and warp directions. The yarns in the direction of loading (Figure 14(d)) show higher damages than the transverse yarns (Figure 14(b)) as expected. Additionally, it can be observed that the damages in the yarns initiate at the crimp zones (see Figures 14 (a) and (c)) which can be attributed to the complexity in geometry resulting in concentrated stresses. Figures 14(e) and (f) show the progressive damage in the matrix. It is to be noted that the matrix damage initiates prior to that of the yarn. Thus, the state of damage in Figure 14(e) is higher than that of yarns in loading direction. This is evident from the macroscopic mechanical response whereby matrix damage initiates at 0.005 . Figures $14(\mathrm{~g})$ and $(\mathrm{h})$ show the current distribution in the unit cell. It is observed that with increasing strain, the overall current flow decreases resulting in an increase of resistivity. It is to be noted that the boundary conditions for the mechanical damage analysis (Figures 14(a-f)) are periodic while the electrical simulations (Figures 14(gh)) are periodic in $\mathrm{Y}$ and $\mathrm{Z}$ directions with a potential gradient applied along $\mathrm{X}$. Overall, the damaged and stressed areas correspond to the resistive zones thus increasing its overall resistivity with increasing strain. 

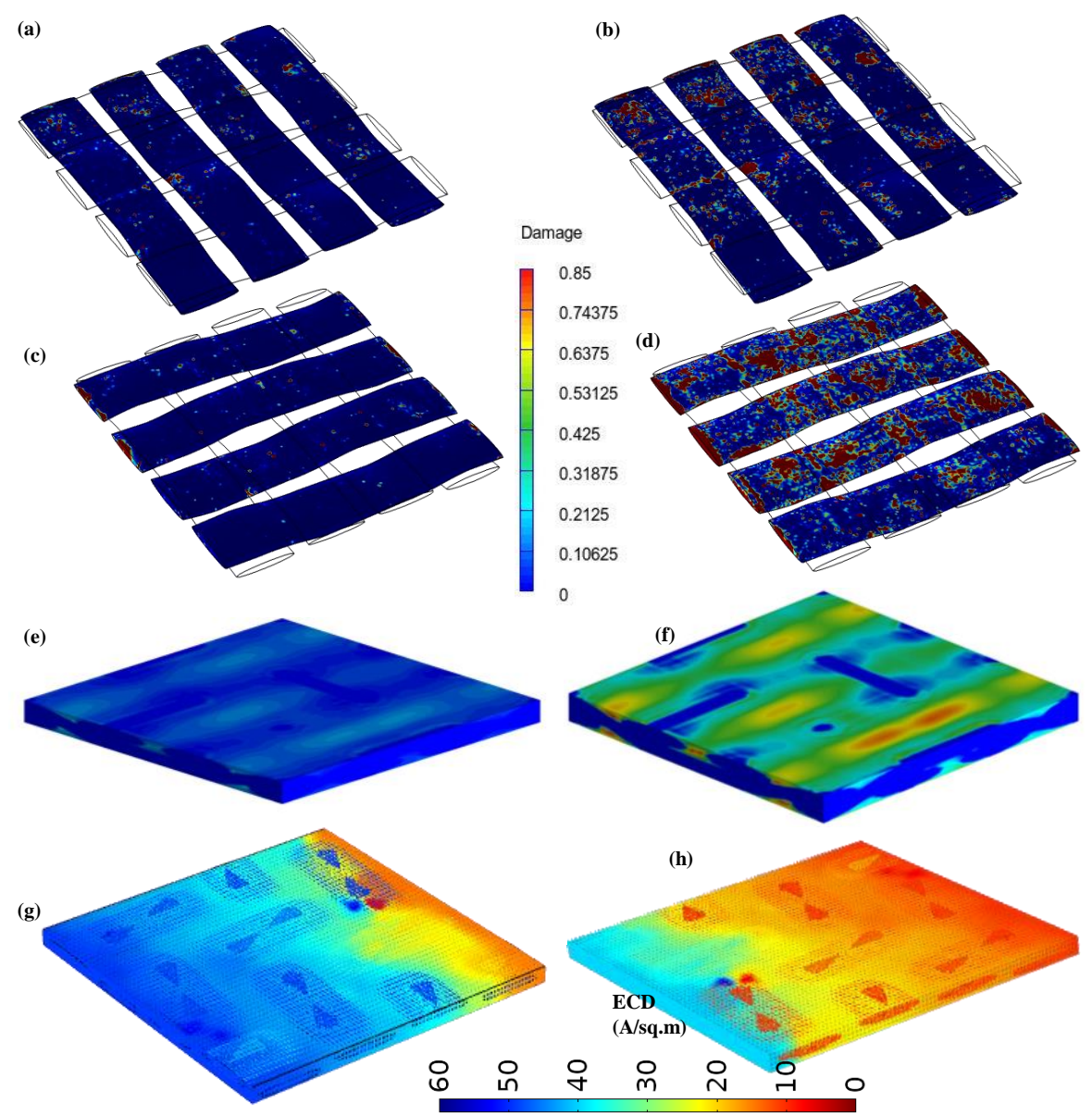

Figure 14. Progressive damage: in weft yarns (at strains of 0.13 (a) and 0.192 (b)) and warp yarns (at strains of 0.13 (c) and 0.192 (d)); Damage progression in CNT-PSS coating matrix corresponding to strains of 0.13 (e) and 0.192 (f) ; Corresponding electrical responses at a strain of $0.13(\mathrm{~g})$ and $0.192(\mathrm{~h})$

Figure 15 shows the FCR with increasing strain for the coated textile weave for tensile loads. While the slight changes in FCR at around 0.005 strain can be attributed to the onset of matrix damage, significant change of slope at 0.085 results from tow debonding. Following the onset of damage in matrix and debonding of yarn, a non-linear response in FCR is observed which correlates with the damage propagation in both the matrix and tows. Beyond a strain of 0.02 , the fibers fail as observed during mechanical simulations which is expected to result in another jump in FCR. However, the FCRs beyond the strain of 0.02 are insignificant in reality owing to the loss of structural integrity of such laminates. 


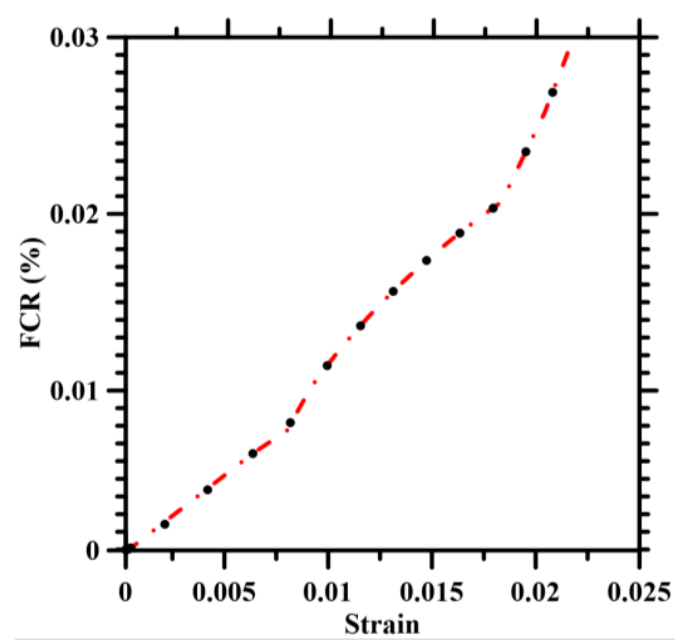

Figure 15. Fractional change in resistance with varying strain for CNT-PSS coated textile weave under tensile load

\subsection{Comparison of simulated responses with experimental observations:}

Figures 16 shows a comparison between the experimental [10] and simulated gauge factors for the smart twill weave laminated composite. The gauge factor [70,71] is a measure of the sensing efficiency and it is expressed as a ratio of the FCR to the corresponding applied strain. Figure 16 shows excellent correlation between the experimental and simulated gauge factors and such good correlation suggests that the multiscale simulation methodology presented in this study can successfully predict the piezoresistive behavior of smart twill weave laminated composite.

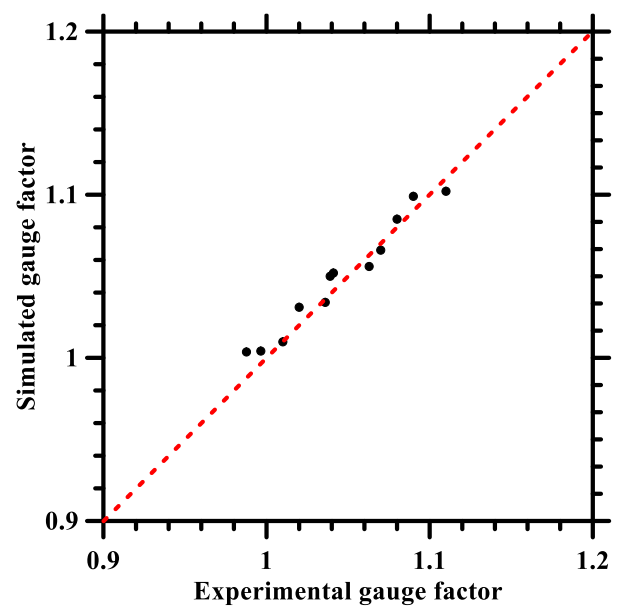

Figure 16. Correlation between experimental [10] and simulated gauge factors 


\section{CONCLUSIONS}

This paper presents a comprehensive multiscale numerical approach towards prediction of electromechanical response of hierarchical smart twill weave laminated composite thin films. The approach, presented in this paper, is unique in its implementation of prediction of electromechanical behavior at the nano-engineered matrix which serves as the reference for the electromechanical simulation of the macro-scale textile in a micromechanics of failure based approach whereby the mechanical damage is computed at the scale of matrix and fiber thus taking into account the nano-scale sensory behavior of such composites. Moreover, the robustness of the approach lies in its ability to capture deformations and large strains at every length scale while taking into account the electrical behavior of the piezoresistive nano-engineered composite. To assess the prediction capability of the simulation methodology, simulated electro-mechanical responses with varying applied strain are compared with experimental responses available in the literature. Simulated responses show excellent correlation with experimental observations. Such good correlation between the experimental and simulated responses provides confidence on the ability of the simulation methodology to represent the complex hierarchical structures of the smart twill weave laminated composite thin film effectively at multiple length scales. Thus, the multiscale numerical simulations presented in this paper can help in developing strategies to tune the hierarchical structures at multiple length scales towards obtaining efficient material design for desired performance.

\section{REFERENCES}

[1] Barbero EJ. Introduction to Composite Materials Design | Taylor \& Francis Group. Taylor \& Francis; 2017.

[2] Adumitroaie A, Barbero EJ. Stiffness and strength prediction for plain weave textile reinforced composites. Mechanics of Advanced Materials and Structures 2012;19.

[3] Böger L, Wichmann MHG, Meyer LO, Schulte K. Load and health monitoring in glass fibre reinforced composites with an electrically conductive nanocomposite epoxy matrix. Composites Science and Technology 2008;68:1886-94. https://doi.org/10.1016/j.compscitech.2008.01.001.

[4] Todo M, Nakamura T, Mada T, Takahashi K. Measurement of dynamic interlaminar fracture toughness of FRP laminates using dynamic displacement measuring apparatus. Advanced Composite Materials 1998;7:285-97. https://doi.org/10.1163/156855198X00200.

[5] Halabe UB, Vasudevan A, Klinkhachorn P, GangaRao HVS. Detection of subsurface defects in fiber reinforced polymer composite bridge decks using digital infrared thermography. Nondestructive Testing and Evaluation 2007;22:155-75. https://doi.org/10.1080/10589750701448381.

[6] Mirmiran Amir, Wei Yunmei. Damage Assessment of FRP-Encased Concrete Using Ultrasonic Pulse Velocity. Journal of Engineering Mechanics 2001;127:126-35. https://doi.org/10.1061/(ASCE)07339399(2001)127:2(126).

[7] Bastianini F, Corradi M, Borri A, Tommaso A di. Retrofit and monitoring of an historical building using "Smart" CFRP with embedded fibre optic Brillouin sensors. Construction and Building Materials 2005;19:525-35. https://doi.org/10.1016/j.conbuildmat.2005.01.004. 
[8] Merzbacher CI, Kersey AD, Friebele EJ. Fiber optic sensors in concrete structures: a review. Smart Mater Struct 1996;5:196-208. https://doi.org/10.1088/0964-1726/5/2/008.

[9] Olin BD, Meeker WQ. Applications of Statistical Methods to Nondestructive Evaluation. Technometrics 1996;38:95-112. https://doi.org/10.1080/00401706.1996.10484451.

[10] Loyola BR, La Saponara V, Loh KJ. In situ strain monitoring of fiber-reinforced polymers using embedded piezoresistive nanocomposites. J Mater Sci 2010;45:6786-98. https://doi.org/10.1007/s10853-010-4775-y.

[11] Loh KJ, Kim J, Lynch JP, Kam NWS, Kotov NA. Multifunctional layer-by-layer carbon nanotubepolyelectrolyte thin films for strain and corrosion sensing. Smart Mater Struct 2007;16:429-438. https://doi.org/10.1088/0964-1726/16/2/022.

[12] Mortensen LP, Ryu DH, Zhao YJ, Loh KJ. Rapid Assembly of Multifunctional Thin Film Sensors for Wind Turbine Blade Monitoring. Key Engineering Materials 2013;569-570:515-22.

[13] Lee BM, Loh KJ. A 2D percolation-based model for characterizing the piezoresistivity of carbon nanotube-based films. J Mater Sci 2015;50:2973-83. https://doi.org/10.1007/s10853-015-8862-y.

[14] Mohd Radzuan NA, Sulong AB, Sahari J. A review of electrical conductivity models for conductive polymer composite. International Journal of Hydrogen Energy 2017;42:9262-73. https://doi.org/10.1016/j.ijhydene.2016.03.045.

[15] Shen Y, He P, Zhuang X. Fracture model for the prediction of the electrical percolation threshold in CNTs/Polymer composites. Front Struct Civ Eng 2018;12:125-36. https://doi.org/10.1007/s11709017-0396-8.

[16] Fleischhauer R, Božić M, Kaliske M. A novel approach to computational homogenization and its application to fully coupled two-scale thermomechanics. Comput Mech 2016;58:769-96. https://doi.org/10.1007/s00466-016-1315-x.

[17] Ullah Z, Kaczmarczyk Ł, Grammatikos SA, Evernden MC, Pearce CJ. Multi-scale computational homogenisation to predict the long-term durability of composite structures. Computers \& Structures 2017;181:21-31. https://doi.org/10.1016/j.compstruc.2016.11.002.

[18] Odegard GM. Constitutive modeling of piezoelectric polymer composites. Acta Materialia 2004;52:5315-30. https://doi.org/10.1016/j.actamat.2004.07.037.

[19] Hallal A, Younes R, Fardoun F. Review and comparative study of analytical modeling for the elastic properties of textile composites. Composites Part B: Engineering 2013;50:22-31. https://doi.org/10.1016/j.compositesb.2013.01.024.

[20] Yang J-M, Ma C-L, Chou T-W. Fiber Inclination Model of Three-Dimensional Textile Structural Composites. Journal of Composite Materials 1986;20:472-84. https://doi.org/10.1177/002199838602000505.

[21] Jacques S, De Baere I, Van Paepegem W. Application of periodic boundary conditions on multiple part finite element meshes for the meso-scale homogenization of textile fabric composites. Composites Science and Technology 2014;92:41-54. https://doi.org/10.1016/j.compscitech.2013.11.023.

[22] Gager J, Pettermann HE. Numerical homogenization of textile composites based on shell element discretization. Composites Science and Technology 2012;72:806-12. https://doi.org/10.1016/j.compscitech.2012.02.009.

[23] Hill R. On constitutive macro-variables for heterogeneous solids at finite strain. Proceedings of the Royal Society of London A Mathematical and Physical Sciences 1972;326:131-47. https://doi.org/10.1098/rspa.1972.0001. 
[24] Zhou X-Y, Gosling PD, Pearce CJ, Ullah Z, Kaczmarczyk L. Perturbation-based stochastic multiscale computational homogenization method for woven textile composites. International Journal of Solids and Structures 2016;80:368-80. https://doi.org/10.1016/j.ijsolstr.2015.09.008.

[25] Kaczmarczyk Ł, Pearce CJ, Bićanić N. Scale transition and enforcement of RVE boundary conditions in second-order computational homogenization. International Journal for Numerical Methods in Engineering 2008;74:506-22. https://doi.org/10.1002/nme.2188.

[26] Keip M-A, Steinmann P, Schröder J. Two-scale computational homogenization of electro-elasticity at finite strains. Computer Methods in Applied Mechanics and Engineering 2014;278:62-79. https://doi.org/10.1016/j.cma.2014.04.020.

[27] Nayak S, Das S. A microstructure-guided numerical approach to evaluate strain sensing and damage detection ability of random heterogeneous self-sensing structural materials. Computational Materials Science 2019;156:195-205.

[28] Song W, Krishnaswamy V, Pucha RV. Computational homogenization in RVE models with material periodic conditions for CNT polymer composites. Composite Structures 2016;137:9-17. https://doi.org/10.1016/j.compstruct.2015.11.013.

[29] Nayak S, Krishnan NMA, Das S. Fracture response of metallic particulate-reinforced cementitious composites: Insights from experiments and multiscale numerical simulations. Cement and Concrete Composites 2019;97:154-65.

[30] Nayak S, Krishnan NMA, Das S. Microstructure-guided numerical simulation to evaluate the influence of phase change materials (PCMs) on the freeze-thaw response of concrete pavements. Construction and Building Materials 2019;201:246-56.

[31] Zaïri F, Naït-Abdelaziz M, Gloaguen JM, Lefebvre JM. Modelling of the elasto-viscoplastic damage behaviour of glassy polymers. International Journal of Plasticity 2008;24:945-65. https://doi.org/10.1016/j.ijplas.2007.08.001.

[32] Bodner SR, Partom Y. Constitutive Equations for Elastic-Viscoplastic Strain-Hardening Materials. J Appl Mech 1975;42:385-9. https://doi.org/10.1115/1.3423586.

[33] Gurson AL. Continuum Theory of Ductile Rupture by Void Nucleation and Growth: Part I-Yield Criteria and Flow Rules for Porous Ductile Media. J Eng Mater Technol 1977;99:2-15. https://doi.org/10.1115/1.3443401.

[34] Pan J, Saje M, Needleman A. Localization of deformation in rate sensitive porous plastic solids. Int J Fract 1983;21:261-78. https://doi.org/10.1007/BF00942345.

[35] Kaseem M, Hamad K, Ko YG. Fabrication and materials properties of polystyrene/carbon nanotube (PS/CNT) composites: A review. European Polymer Journal 2016;79:36-62. https://doi.org/10.1016/j.eurpolymj.2016.04.011.

[36] Nouri N, Ziaei-Rad S, Adibi S, Karimzadeh F. Fabrication and mechanical property prediction of carbon nanotube reinforced Aluminum nanocomposites. Materials \& Design 2012;34:1-14. https://doi.org/10.1016/j.matdes.2011.07.047.

[37] Doner S, Nayak S, Senol K, Shukla A, Krishnan NMA, Yilmazcoban IK, et al. Dynamic compressive behavior of metallic particulate-reinforced cementitious composites: SHPB experiments and numerical simulations. Construction and Building Materials 2019;227:116668. https://doi.org/10.1016/j.conbuildmat.2019.08.049.

[38] Ortiz M, Pandolfi A. Finite-deformation irreversible cohesive elements for three-dimensional crackpropagation analysis. International Journal for Numerical Methods in Engineering 1999;44:1267-82. 
[39] Wu T, Wriggers P. Multiscale diffusion-thermal-mechanical cohesive zone model for concrete. Computational Mechanics 2015;55:999-1016.

[40] Li D, Yang Q-S, Liu X, He X-Q. Experimental and cohesive finite element investigation of interfacial behavior of CNT fiber-reinforced composites. Composites Part A: Applied Science and Manufacturing 2017;101:318-25. https://doi.org/10.1016/j.compositesa.2017.06.033.

[41] Chen X, Zhang L, Zheng M, Park C, Wang X, Ke C. Quantitative nanomechanical characterization of the van der Waals interfaces between carbon nanotubes and epoxy. Carbon 2015;82:214-28. https://doi.org/10.1016/j.carbon.2014.10.065.

[42] Namilae S, Chandra N. Multiscale Model to Study the Effect of Interfaces in Carbon Nanotube-Based Composites | Journal of Engineering Materials and Technology | ASME Digital Collection. Journal of Engineering Materials and Technology 2005;127:222-32.

[43] Quaresimin M, Salviato M, Zappalorto M. A multi-scale and multi-mechanism approach for the fracture toughness assessment of polymer nanocomposites. Composites Science and Technology 2014;91:16-21. https://doi.org/10.1016/j.compscitech.2013.11.015.

[44] Quaresimin M, Schulte K, Zappalorto M, Chandrasekaran S. Toughening mechanisms in polymer nanocomposites: From experiments to modelling. Composites Science and Technology 2016;123:187-204. https://doi.org/10.1016/j.compscitech.2015.11.027.

[45] Hsieh TH, Kinloch AJ, Taylor AC, Kinloch IA. The effect of carbon nanotubes on the fracture toughness and fatigue performance of a thermosetting epoxy polymer. Journal of Materials Science 2011;46:7525.

[46] Quaresimin M, Schulte K, Zappalorto M, Chandrasekaran S. Toughening mechanisms in polymer nanocomposites: From experiments to modelling. Composites Science and Technology 2016;123:187-204. https://doi.org/10.1016/j.compscitech.2015.11.027.

[47] Marshall GP, Culver LE, Williams JG. Fracture phenomena in polystyrene. Int J Fract 1973;9:295309. https://doi.org/10.1007/BF00049197.

[48] Ayatollahi MR, Aliha MRM. On determination of mode II fracture toughness using semi-circular bend specimen. International Journal of Solids and Structures 2006;43:5217-27. https://doi.org/10.1016/j.ijsolstr.2005.07.049.

[49] Hu B, Hu N, Li Y, Akagi K, Yuan W, Watanabe T, et al. Multi-scale numerical simulations on piezoresistivity of CNT/polymer nanocomposites. Nanoscale Research Letters 2012;7:402. https://doi.org/10.1186/1556-276X-7-402.

[50] Kirkpatrick S. Percolation and Conduction. Rev Mod Phys 1973;45:574-88. https://doi.org/10.1103/RevModPhys.45.574.

[51] Lesieutre GA. Finite elements for dynamic modeling of uniaxial rods with frequency-dependent material properties. International Journal of Solids and Structures 1992;29:1567-79. https://doi.org/10.1016/0020-7683(92)90134-F.

[52] Wang L, Wu J, Chen C, Zheng C, Li B, Joshi SC, et al. Progressive failure analysis of 2D woven composites at the meso-micro scale. Composite Structures 2017;178:395-405. https://doi.org/10.1016/j.compstruct.2017.07.023.

[53] Tang X, Whitcomb JD. Progressive Failure Behaviors of 2D Woven Composites. Journal of Composite Materials 2003;37:1239-59. https://doi.org/10.1177/0021998303037014002.

[54] Lin H, Brown LP, Long AC. Modelling and Simulating Textile Structures Using TexGen. Advanced Materials Research 2011. https://doi.org/10.4028/www.scientific.net/AMR.331.44. 
[55] Li S. Boundary conditions for unit cells from periodic microstructures and their implications. Composites $\quad$ Science 2008;68:1962-74. https://doi.org/10.1016/j.compscitech.2007.03.035.

[56] Liu H, Zeng D, Li Y, Jiang L. Development of RVE-embedded solid elements model for predicting effective elastic constants of discontinuous fiber reinforced composites. Mechanics of Materials 2016;93:109-23. https://doi.org/10.1016/j.mechmat.2015.10.011.

[57] Xia Z, Zhou C, Yong Q, Wang X. On selection of repeated unit cell model and application of unified periodic boundary conditions in micro-mechanical analysis of composites. International Journal of Solids and Structures 2006;43:266-78. https://doi.org/10.1016/j.ijsolstr.2005.03.055.

[58] Li X, Binienda WK, Goldberg Robert K. Finite-Element Model for Failure Study of TwoDimensional Triaxially Braided Composite. Journal of Aerospace Engineering 2011;24.

[59] Jiang H, Ren Y, Zhang S, Liu Z, Nie L. Multi-scale finite element analysis for tension and ballistic penetration damage characterizations of 2D triaxially braided composite. J Mater Sci 2018;53:1007194. https://doi.org/10.1007/s10853-018-2248-x.

[60] Zhang C, Li N, Wang W, Binienda WK, Fang H. Progressive damage simulation of triaxially braided composite using a 3D meso-scale finite element model. Composite Structures 2015;125:104-16. https://doi.org/10.1016/j.compstruct.2015.01.034.

[61] Zeng Q, Sun L, Ge J, Wu W, Liang J, Fang D. Damage characterization and numerical simulation of shear experiment of plain woven glass-fiber reinforced composites based on 3D geometric reconstruction. Composite Structures 2020;233:111746. https://doi.org/10.1016/j.compstruct.2019.111746.

[62] Pegoretti A, Accorsi ML, Dibenedetto AT. Fracture toughness of the fibre-matrix interface in glassepoxy composites. JOURNAL OF MATERIALS SCIENCE 1996;31:6145-53. https://doi.org/10.1007/BF00354431.

[63] Lou X, Cai H, Yu P, Jiao F, Han X. Failure analysis of composite laminate under low-velocity impact based on micromechanics of failure. Composite Structures 2017;163:238-47. https://doi.org/10.1016/j.compstruct.2016.12.030.

[64] Wang L, Zheng C, Wei S, Wei Z. Micromechanics-based progressive failure analysis of carbon fiber/epoxy composite vessel under combined internal pressure and thermomechanical loading. Composites Part B: Engineering 2016;89:77-84. https://doi.org/10.1016/j.compositesb.2015.11.018.

[65] Bažant ZP, Oh BH. Crack band theory for fracture of concrete. Mat Constr 1983;16:155-77. https://doi.org/10.1007/BF02486267.

[66] Benzeggagh ML, Kenane M. Measurement of mixed-mode delamination fracture toughness of unidirectional glass/epoxy composites with mixed-mode bending apparatus. Composites Science and Technology 1996;56:439-49. https://doi.org/10.1016/0266-3538(96)00005-X.

[67] Herráez M, Fernández A, Lopes CS, González C. Strength and toughness of structural fibres for composite material reinforcement. Philosophical Transactions of the Royal Society A: Mathematical, Physical and Engineering Sciences 2016;374:20150274. https://doi.org/10.1098/rsta.2015.0274.

[68] Arbaoui J, Tarfaoui M, El Malki Alaoui A. Mechanical behavior and damage kinetics of woven Eglass/vinylester laminate composites under high strain rate dynamic compressive loading: Experimental and numerical investigation. International Journal of Impact Engineering 2016;87:4454. https://doi.org/10.1016/j.ijimpeng.2015.06.026.

[69] Callinan TD. The Electrical Properties of Glass Fiber Paper - II. Naval Research Laboratory; 1952. 
[70] Yang P, Chowdhury S, Neithalath N. Strain sensing ability of metallic particulate reinforced cementitious composites: Experiments and microstructure-guided finite element modeling. Cement and Concrete Composites 2018;90:225-34.

[71] Chung DDL. Piezoresistive cement-based materials for strain sensing. Journal of Intelligent Material Systems and Structures 2002;13:599-609. 